\title{
Zero-jettiness resummation for top-quark pair production at the LHC
}

\author{
Simone Alioli, ${ }^{a}$ Alessandro Broggio ${ }^{a}$ and Matthew A. Lim ${ }^{a, b}$ \\ ${ }^{a}$ Università degli Studi di Milano-Bicocca $\mathscr{G}$ INFN, \\ Piazza della Scienza 3, Milano 20126, Italy \\ ${ }^{b}$ Deutsches Elektronen-Synchrotron DESY, \\ Notkestr. 85, 22607 Hamburg, Germany \\ E-mail: simone.alioli@unimib.it, alessandro.broggio@unimib.it, \\ matthew.lim@desy.de
}

ABSTRACT: We study the resummation of the 0-jettiness resolution variable $\mathcal{T}_{0}$ for the top-quark pair production process in hadronic collisions. Starting from an effective theory framework we derive a factorisation formula for this observable which allows its resummation at any logarithmic order in the $\mathcal{T}_{0} \rightarrow 0$ limit. We then calculate the $\mathcal{O}\left(\alpha_{s}\right)$ corrections to the soft function matrices and, by employing renormalisation group equation methods, we obtain the ingredients for the resummation formula up to next-to-next-to-leading logarithmic (NNLL) accuracy. We study the impact of these corrections to the 0-jettiness distribution by comparing predictions at different accuracy orders: NLL, NLL', NNLL and approximate NNLL' (NNLL $L_{\mathrm{a}}^{\prime}$ ). We match these results to the corresponding fixed order calculations both at leading order and next-to-leading order for the $t \bar{t}+$ jet production process, obtaining the most accurate prediction of the 0-jettiness distribution for the top-quark pair production process at $\mathrm{NNLL}_{\mathrm{a}}^{\prime}+\mathrm{NLO}$ accuracy.

KeYwords: NLO Computations, QCD Phenomenology

ARXIV EPRINT: 2111.03632 


\section{Contents}

1 Introduction 1

2 Zero-jettiness factorisation for top-quark pair production 4

2.1 Process definition 4

2.2 Factorisation formula for top-quark pair production 5

2.3 Factorisation theorem in Laplace space 6

$\begin{array}{ll}2.4 & \text { The hard function and its evolution }\end{array}$

2.5 The soft function and its evolution 8

2.5.1 Calculation of the one-loop soft function 8

2.5.2 Solving the soft RG equations at fixed order 9

$\begin{array}{ll}2.5 .3 \text { Evolution } & 10\end{array}$

$\begin{array}{lll}2.6 & \text { The beam functions and their evolution } & 11\end{array}$

3 Resummation via renormalisation group evolution $\quad \mathbf{1 2}$

$\begin{array}{lll}3.1 & \text { All-order solutions of the RG equations } & 12\end{array}$

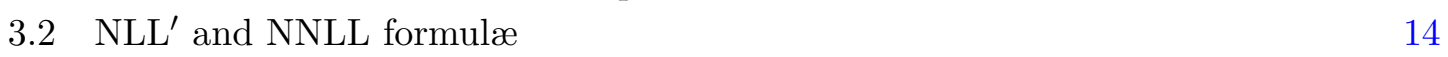

$\begin{array}{lll}3.3 & \text { NNLL' formulæ } & 16\end{array}$

$\begin{array}{lll}4 & \text { Numerical results } & 17\end{array}$

5 Conclusions 21

A Derivation of the factorisation formula 23

B One-loop soft integrals $\quad 31$

B.1 Heavy-heavy integrals, same leg 32

B.2 Heavy-heavy integrals, opposite leg 32

B.3 Heavy-light integrals, same hemisphere 33

B.4 Heavy-light integrals, opposite hemisphere 33

\section{Introduction}

Top-quark pair production is a process of enormous interest at the LHC and at future hadron colliders. The top-quark is the most massive of the Standard Model (SM) particles and couples strongly to the Higgs boson - studies of its properties are therefore vital in order to test the SM at the precision frontier and probe the nature of the observed Higgs sector. The top sector is also of interest from the perspective of constraining higher dimensional operators in the Standard Model Effective Theory, as well as for fitting parton distribution functions. For all of these purposes, it is necessary to have predictions for the main top-quark production channel, i.e. pair production, at the highest possible accuracy. 
There is an abundance of experimental measurements of the $t \bar{t}$ process at all LHC run energies and with a range of luminosities, for example refs. [1-11]. Such measurements are important for extracting properties of the SM such as the strong coupling $\alpha_{s}$ and the top-quark mass $[12,13]$.

The first predictions for the $t \bar{t}$ total cross section at next-to-next-to-leading order (NNLO) in quantum chromodynamics (QCD) appeared in refs. [14-16]. These were followed by predictions for differential distributions of stable top-quarks computed in the STRIPPER local subtraction framework [17-20]. The two-loop amplitudes computed for the aforementioned calculations were later reused in a new calculation at NNLO via the $q_{T}$-slicing approach [21-23]. NNLO corrections to both top-quark production and decay were combined in the narrow-width approximation in refs. [24, 25], while NLO electroweak (EW) corrections (first computed in refs. [26-28]) were combined with NNLO QCD corrections for stable tops in ref. [29].

In addition to calculations at fixed order (FO) in perturbation theory, resummed calculations have also appeared for a variety of observables for this process. Following the computation of the relevant anomalous dimensions in refs. [30, 31], threshold resummation at next-to-next-to-leading logarithmic accuracy (NNLL) was achieved via Soft-Collinear Effective Theory (SCET). This was carried out in different kinematic regimes - the pair invariant mass kinematics were studied in ref. [32], while the treatment of the 1-particle inclusive kinematics appeared in ref. [33]. Within this paradigm, more differential results including approximate NNLO corrections to the production and NLO (NNLO) corrections to the decay appeared in refs. [34, 35], while studies of boosted top-quark production matched to both NLO and NNLO calculations were carried out in refs. [36, 37]. The transverse momentum of the $t \bar{t}$ pair was resummed within SCET in ref. [38], while the framework for performing the same resummation in direct QCD was later established in ref. [39].

Recently, NNLO predictions were matched to a parton shower in ref. [40] using the MINNLO $_{P S}$ method [41], allowing the generation of events at NNLO+PS accuracy. This work utilised the factorisation theorem of ref. [39] and was made possible by the calculation of the necessary perturbative ingredients in ref. [21] as well as the POWHEG implementations of the $t \bar{t}$ and $t \bar{t}+$ jet processes $[42,43]$.

In this work, we develop a resummation framework using the 0 -jettiness variable $\mathcal{T}_{0}$ for the production of a $t \bar{t}$ pair at the LHC, which is a required ingredient for the creation of a NNLO+PS event generator in the GENEva formalism [44] when using $\mathcal{T}_{0}$ as resolution variable. ${ }^{1}$

The $N$-jettiness variable $\mathcal{T}_{N}$ was first introduced in ref. [47] as a means by which to discriminate between resolved emissions with different jet multiplicities. Given an $M$-particle phase space point $\Phi_{M}$ with $M \geq N$, it is defined as

$$
\mathcal{T}_{N}\left(\Phi_{M}\right)=\sum_{k} \min \left\{\hat{q}_{a} \cdot p_{k}, \hat{q}_{b} \cdot p_{k}, \hat{q}_{1} \cdot p_{k}, \ldots, \hat{q}_{N} \cdot p_{k}\right\}
$$

\footnotetext{
${ }^{1}$ Recently the Geneva framework has also been implemented using the transverse momentum of the colour singlet as resolution variable for the DY production process [45]. Given that the factorisation theorem for the transverse momentum of the $t \bar{t}$ pair has already been established in ref. [38] and the relevant soft functions have been calculated at two-loop order in ref. [46], one can envision a similar development also for $t \bar{t}$ production. We leave this extension to future work.
} 
where $\hat{q}_{i}=n_{i}=\left(1, \vec{n}_{i}\right)$ are light-like reference vectors parallel to the beam and jet directions, defined in the rest frame of the Born-level final state, and the index $k$ runs over all stronglyinteracting final-state particles. The limit $\mathcal{T}_{N} \rightarrow 0$ describes an event with $N$ pencil-like hard jets, where the unresolved emissions can either be soft or collinear to the final-state jets or to the beams. ${ }^{2}$

A factorisation theorem for the 0-jettiness, valid for colour-singlet production, was derived in ref. [48] via SCET methods. This allowed the resummation of logarithms of $\mathcal{T}_{0} / Q$ for some hard scale $Q$, and has been the basis for the majority of practical implementations of the Geneva framework thus far. In the Geneva approach, a resummed calculation in a suitable resolution variable is combined with the appropriate fixed order predictions and matched to a parton shower, thus providing accurate predictions across the whole phase space. The method has been successfully applied to several processes involving colour-singlet particles in the final state (or coloured particles produced from decaying colour singlets) up to $\mathrm{N}^{3} \mathrm{LL}+\mathrm{NNLO}$ accuracy $[45,49-53]$. This work paves the way for the first Geneva implementation for the production of massive coloured particles in hadronic collisions.

The $N$-jettiness can also be used as a slicing parameter for fixed order calculations [54]. In particular, a 0-jettiness subtraction has been used to obtain NNLO predictions for an array of colour-singlet production processes in MCFM [55, 56]. The method has also recently been extended to $\mathrm{N}^{3} \mathrm{LO}$ in ref. [57]. The results presented in this work provide an avenue towards a future application of the 0-jettiness subtraction to the production of heavy quark pairs at NNLO accuracy.

In addition to its intrinsic utility for the GENEvA or MCFM implementations, 0-jettiness is an important observable to disentangle the effects of underlying-event and multi-parton interactions in hadronic collisions. The reason is that contributions from particles in the forward and backward directions are suppressed with respect to particles emitted at central pseudorapidities. Therefore, measuring 0-jettiness, or beam-thrust [58], and comparing it with other observables like the scalar sum of all transverse momenta gives a handle on the hadronic activity from initial-state radiation.

When using the $N$-jettiness as a resolution variable for processes involving massive coloured particles, there are two possible choices one could make. The first option is to extend the definition in eq. (1.1) to include the velocities of the top-quarks as additional reference directions, i.e. to supplement the light-like $\hat{q}_{i}$ with two additional massive vectors. This is similar to the approach taken in refs. [59-61], which also resums logarithms arising from radiation collinear to the heavy quarks. This is the appropriate treatment when the top-quarks are in the boosted regime, with the top-quark pair invariant mass $M \gg m_{t}$.

Alternatively, one could exclude the final-state top-quarks from the sum in eq. (1.1). In this way, additional radiation is never clustered together with a top-quark, which ultimately is not a stable particle. In the soft and collinear limit and provided that the top-quarks are

\footnotetext{
${ }^{2}$ Different procedures can be devised to identify the jet directions $\hat{q}_{i}$ that truly minimise the sum - for example, one can identify the jet directions with the axis found by a jet clustering algorithm. The differences between these alternative definitions are usually of nonsingular nature. Given that the only directions which are important for $\mathcal{T}_{0}$ are those of the beams and there is no ambiguity in their definition, we will not be concerned with this issue for the rest of this paper.
} 
not highly boosted, the two choices are equivalent, in the sense that all singularities are captured as $\mathcal{T}_{N} \rightarrow 0$ in both cases. ${ }^{3}$ In this work we make the second of the two choices, and resum the 0 -jettiness so-defined for the $t \bar{t}$ process.

The paper is organised as follows. In section 2, we discuss the factorisation theorem in SCET and the calculation of the necessary perturbative ingredients. We then examine the resummation of logarithms via renormalisation group (RG) evolution in section 3 and provide expressions for the resummed $\mathcal{T}_{0}$ distribution at various resummation orders. We present numerical results for the resummed $\mathcal{T}_{0}$ distribution in section 4 , and finally report our conclusions in section 5 .

\section{Zero-jettiness factorisation for top-quark pair production}

In this section we discuss the factorisation theorem for $\mathcal{T}_{0}$ and the calculation of the perturbative ingredients necessary to achieve NNLL and approximate NNLL' accuracy. We present the factorisation theorem for the $t \bar{t}$ process, whose complete derivation is detailed in appendix A. We also report on the calculation of the relevant soft function at one-loop order, which is a necessary ingredient beginning at NLL' accuracy.

\subsection{Process definition}

We consider the creation of a $t \bar{t}$ pair from the collision of two protons in the process

$$
P_{1}\left(P_{a}\right)+P_{2}\left(P_{b}\right) \rightarrow t\left(p_{3}\right)+\bar{t}\left(p_{4}\right)+X\left(p_{X}\right)
$$

which receives contributions from the quark and gluon-initiated Born level partonic channels

$$
\begin{aligned}
& q\left(p_{1}\right)+\bar{q}\left(p_{2}\right) \rightarrow t\left(p_{3}\right)+\bar{t}\left(p_{4}\right) \quad+\quad(q \leftrightarrow \bar{q}), \\
& g\left(p_{1}\right)+g\left(p_{2}\right) \rightarrow t\left(p_{3}\right)+\bar{t}\left(p_{4}\right)
\end{aligned}
$$

where $p_{1}=x_{1} P_{a}, p_{2}=x_{2} P_{b}$. The kinematics of the process are fully specified by the invariants

$$
\begin{aligned}
& S=\left(P_{a}+P_{b}\right)^{2}, \quad s=x_{1} x_{2} S, \quad M^{2}=\left(p_{3}+p_{4}\right)^{2}, \\
& t_{1}=\left(p_{1}-p_{3}\right)^{2}-m_{t}^{2}, \quad u_{1}=\left(p_{1}-p_{4}\right)^{2}-m_{t}^{2} \text {. }
\end{aligned}
$$

It is convenient to work with two additional variables: the velocity of the top-quarks in the $t \bar{t}$ rest frame $\beta_{t}$ and the scattering angle $\theta$ of the top-quark in the centre-of-mass frame of the event. These can be expressed via the invariants above as

$$
\beta_{t}=\sqrt{1-\frac{4 m_{t}^{2}}{M^{2}}}, \cos \theta=\frac{1}{\beta_{t}}\left(1+\frac{2 t_{1}}{M^{2}}\right) .
$$

\footnotetext{
${ }^{3}$ In this regime, emissions collinear to the top-quark are not divergent. The large quark mass acts as a cut-off, preventing any potential enhancement.
} 


\subsection{Factorisation formula for top-quark pair production}

In order to fully appreciate the ingredients appearing in the factorisation of 0-jettiness for heavy quark pair production, it is instructive to begin by examining the structure of the factorisation theorem for $\mathcal{T}_{0}$ in the case of colour-singlet production, which was first derived in ref. [48]. Defining $\tau_{B} \equiv \mathcal{T}_{0} / M$, with $M$ the mass of the colour singlet object, one can write the factorisation theorem, differential in the Born phase space $\Phi_{0}$, as

$$
\frac{\mathrm{d} \sigma}{\mathrm{d} \Phi_{0} \mathrm{~d} \tau_{B}}=M \sum_{i, j} H_{i j}\left(\Phi_{0}, \mu\right) \int \mathrm{d} t_{a} \mathrm{~d} t_{b} B_{i}\left(t_{a}, z_{a}, \mu\right) B_{j}\left(t_{b}, z_{b}, \mu\right) S_{B}\left(M \tau_{B}-\frac{t_{a}+t_{b}}{M}, \mu\right),
$$

where the objects appearing in this formula are UV-renormalised at a common scale $\mu$. The differential cross section has been separated into hard, soft and beam pieces, each of which is free from large logarithms when it is evaluated at its own characteristic scale. The hard functions $H_{i j}$ arise from the matching of the effective theory onto QCD, and describes the hard dynamics of the specific process under consideration. ${ }^{4}$ The beam functions $B_{i, j}$ describe collinear radiation from the initial state partons which forms, in the language of ref. [48], an 'initial-state jet' with virtuality $t_{a, b}$ and light-cone momentum fraction $z_{a, b}$. These functions are labelled by the flavour of the incoming parton and are process-independent: their perturbative parts were computed up to two-loop order for quarks in refs. [62, 63] and for gluons in refs. [64, 65], and were extended to three-loop order in ref. [66]. Finally, the soft function $S_{B}$ describes the accompanying soft radiation. It was calculated for colour-singlet processes at two-loop order in refs. $[67,68]$ and remains unknown at three-loop order. The objects discussed here all have operatorial definitions in SCET, which can be found in e.g. refs. [48, 62].

The analogous factorisation theorem for the $t \bar{t}$ process may be derived using SCET and heavy-quark effective theory (HQET). We present its derivation in appendix A: the final result reads

$$
\begin{aligned}
\frac{\mathrm{d} \sigma}{\mathrm{d} \Phi_{0} \mathrm{~d} \tau_{B}}=M \sum_{i, j} & \int \mathrm{d} t_{a} \mathrm{~d} t_{b} B_{i}\left(t_{a}, z_{a}, \mu\right) B_{j}\left(t_{b}, z_{b}, \mu\right) \\
& \times \operatorname{Tr}\left\{\mathbf{H}_{i j}\left(\Phi_{0}, \mu\right) \mathbf{S}_{B, i j}\left(M \tau_{B}-\frac{t_{a}+t_{b}}{M}, \Phi_{0}, \mu\right)\right\},
\end{aligned}
$$

where the sum runs over the possible channels $i, j=\{q \bar{q}, \bar{q} q, g g\}$, including all light quark flavours, and $M$ is the top-quark pair invariant mass. The charge-conjugated quark-initiated channels give equivalent contributions at leading order but they start to differ at higher orders, leading to the well-known charge asymmetry in top-quark pair production.

The main difference compared to the colour singlet production case is that the hard and soft functions are now matrices in colour space. The soft functions also fully depend on the LO kinematics of the process. ${ }^{5}$ The beam functions remain process-independent objects and have the same definition as in the colour singlet case.

\footnotetext{
${ }^{4}$ In order to be completely general over colour singlet processes, we have included the flux factor and other process-specific prefactors within the hard functions in our treatment.

${ }^{5}$ Following its operatorial definition in eq. (A.30) the soft function contains also a nonperturbative component, which is usually included via dedicated models in resummed calculations. For the rest of this
} 
Despite its apparent simplicity, eq. (2.6) is an important result of this paper. It establishes a framework for the resummation of $\tau_{B}$, fully differential in the Born phase space, which is valid at any logarithmic order near the soft or collinear limits where $\tau_{B} \ll 1$.

\subsection{Factorisation theorem in Laplace space}

Proceeding with the evaluation of eq. (2.6) in momentum space is rather tedious, since the ingredients of the factorisation theorem are distribution-valued and are tied together by convolutions. In Laplace space, however, each object can be expressed in terms of logarithms of the conjugate variable and the theorem is written as a product rather than a convolution. This simplifies the renormalisation of the soft function and the consistency checks between the anomalous dimensions, and allows one to solve the evolution equations as ordinary differential equations. Once all solutions have been found, the inverse transform can be applied to the resummed pieces which are then combined to obtain the correct expression in momentum space.

Taking the Laplace transform of eq. (2.6) with respect to $M \tau_{B}$, therefore, we find that (suppressing some of the arguments of the functions for brevity)

$$
\begin{gathered}
\mathcal{L}\left[\frac{\mathrm{d} \sigma}{\mathrm{d} \Phi_{0} \mathrm{~d} \tau_{B}}\right]= \\
M \int \mathrm{d}\left(M \tau_{B}\right) e^{-\lambda M \tau_{B}} \int \mathrm{d} t_{a} \mathrm{~d} t_{b} B_{i}\left(t_{a}\right) B_{j}\left(t_{b}\right) \operatorname{Tr}\left\{\mathbf{H}_{i j} \mathbf{S}_{B, i j}\left(M \tau_{B}-\frac{t_{a}+t_{b}}{M}\right)\right\} \\
=M \int \mathrm{d} \Omega e^{-\lambda \Omega} \operatorname{Tr}\left\{\mathbf{H}_{i j} \mathbf{S}_{B, i j}(\Omega)\right\} \int \mathrm{d} t_{a} e^{-\frac{\lambda t_{a}}{M}} B_{i}\left(t_{a}\right) \int \mathrm{d} t_{b} e^{-\frac{\lambda t_{b}}{M}} B_{j}\left(t_{b}\right),
\end{gathered}
$$

where we have defined $\Omega \equiv M \tau_{B}-\left(t_{a}+t_{b}\right) / M$ and the Laplace transform is identified by $\mathcal{L}[\ldots]$. Using the definitions of the Laplace transforms of the beam $\tilde{B}=\mathcal{L}[B]$ and soft $\tilde{\mathbf{S}}=\mathcal{L}[\mathbf{S}]$ functions and defining $\kappa=e^{-\gamma_{E}} / \lambda$, we finally arrive at

$$
\begin{aligned}
\mathcal{L}\left[\frac{\mathrm{d} \sigma}{\mathrm{d} \Phi_{0} \mathrm{~d} \tau_{B}}\right] & =M \operatorname{Tr}\left\{\mathbf{H}_{i j} \tilde{\mathbf{S}}_{B, i j}\left(\ln \frac{1}{\left(\lambda e^{\gamma_{E}} \mu\right)^{2}}\right)\right\} \tilde{B}_{i}\left(\ln \left(\frac{M}{\lambda \mu^{2} e^{\gamma_{E}}}\right)\right) \tilde{B}_{j}\left(\ln \left(\frac{M}{\lambda \mu^{2} e^{\gamma_{E}}}\right)\right) \\
& =M \operatorname{Tr}\left\{\mathbf{H}_{i j} \tilde{\mathbf{S}}_{B, i j}\left(\ln \frac{\kappa^{2}}{\mu^{2}}\right)\right\} \tilde{B}_{i}\left(\ln \frac{M \kappa}{\mu^{2}}\right) \tilde{B}_{j}\left(\ln \frac{M \kappa}{\mu^{2}}\right) .
\end{aligned}
$$

We have thus been able to express the differential cross section as a product of functions in Laplace space. Moreover, the Laplace-transformed soft function in eq. (2.8) can be written as a polynomial in the logarithm of the Laplace variable $\kappa$, with function-valued coefficients.

We are now in a position to solve the evolution equations to all orders and hence perform the resummation. We consider the various ingredients of the factorisation theorem in turn.

\subsection{The hard function and its evolution}

The colour-decomposed hard functions $\mathbf{H}_{i j}\left(\Phi_{0}, \mu\right)$ for $t \bar{t}$ production were first computed at one-loop order in ref. [32]. The two-loop amplitudes which are necessary for the construction

paper, we will only consider the perturbative part of the soft function. After implementing the results of this calculation fully into GENEvA, these nonperturbative effects can be recovered via the hadronisation model in the shower Monte Carlo. 
of the NNLO hard functions can instead be found in ref. [69]. From hereon we express the $\Phi_{0}$ dependence in terms of the variables $\beta_{t}, \theta$ defined in eq. (2.4) and the top-quark pair invariant mass $M$. Dropping the channel subscripts for ease of notation, each hard function satisfies the following RG equation [32]

$$
\frac{\mathrm{d}}{\mathrm{d} \ln \mu} \mathbf{H}\left(M, \beta_{t}, \theta, \mu\right)=\boldsymbol{\Gamma}_{H}\left(M, \beta_{t}, \theta, \mu\right) \mathbf{H}\left(M, \beta_{t}, \theta, \mu\right)+\mathbf{H}\left(M, \beta_{t}, \theta, \mu\right) \boldsymbol{\Gamma}_{H}^{\dagger}\left(M, \beta_{t}, \theta, \mu\right),
$$

where we conveniently wrote the anomalous dimension

$$
\boldsymbol{\Gamma}_{H}\left(M, \beta_{t}, \theta, \mu\right)=\Gamma_{\text {cusp }}\left(\alpha_{s}\right)\left(\ln \frac{M^{2}}{\mu^{2}}-i \pi\right)+\gamma^{h}\left(M, \beta_{t}, \theta, \alpha_{s}\right) .
$$

The non-cusp anomalous dimension matrices $\gamma^{h}$ were computed up to two-loop order in refs. [30, 31]. The all-order solution can be written as [32]

$$
\mathbf{H}\left(M, \beta_{t}, \theta, \mu\right)=\mathbf{U}\left(M, \beta_{t}, \theta, \mu_{h}, \mu\right) \mathbf{H}\left(M, \beta_{t}, \theta, \mu_{h}\right) \mathbf{U}^{\dagger}\left(M, \beta_{t}, \theta, \mu_{h}, \mu\right),
$$

where $\mu_{h}$ is a hard scale of the process, e.g. the $t \bar{t}$ invariant mass $M$, such that the hard function is free from large logarithms. When evaluated at a generic scale $\mu$ instead of at the hard scale $\mu_{h}$, the matrix $\mathbf{U}$ performs the resummation of these hard logarithms.

For later convenience, we use the fact that $\mathbf{U}$ can be rewritten by separating out a part which comes from the cusp evolution and is diagonal in colour space and a leftover piece $\mathbf{u}$ which also contains non-diagonal contributions:

$$
\mathbf{U}\left(M, \beta_{t}, \theta, \mu_{h}, \mu\right)=\exp \left[2 S\left(\mu_{h}, \mu\right)-a_{\Gamma}\left(\mu_{h}, \mu\right)\left(\ln \frac{M^{2}}{\mu_{h}^{2}}-i \pi\right)\right] \mathbf{u}\left(M, \beta_{t}, \theta, \mu_{h}, \mu\right) .
$$

The double and (part of) the single logarithmic resummation are provided by the functions $S$ and $a_{\Gamma}$ respectively, defined as

$$
\begin{aligned}
S\left(\mu_{a}, \mu_{b}\right) & =-\int_{\alpha_{s}\left(\mu_{a}\right)}^{\alpha_{s}\left(\mu_{b}\right)} \mathrm{d} \alpha \frac{\Gamma_{\text {cusp }}(\alpha)}{\beta(\alpha)} \int_{\alpha_{s}\left(\mu_{a}\right)}^{\alpha} \frac{\mathrm{d} \alpha^{\prime}}{\beta\left(\alpha^{\prime}\right)}, \\
a_{\Gamma}\left(\mu_{a}, \mu_{b}\right) & =-\int_{\alpha_{s}\left(\mu_{a}\right)}^{\alpha_{s}\left(\mu_{b}\right)} \mathrm{d} \alpha \frac{\Gamma_{\text {cusp }}(\alpha)}{\beta(\alpha)} .
\end{aligned}
$$

The off-diagonal, non-cusp evolution is instead provided by the colour matrix

$$
\mathbf{u}\left(M, \beta_{t}, \theta, \mu_{h}, \mu\right)=\mathcal{P} \exp \int_{\alpha_{s}\left(\mu_{h}\right)}^{\alpha_{s}(\mu)} \frac{\mathrm{d} \alpha}{\beta(\alpha)} \gamma^{h}\left(M, \beta_{t}, \theta, \alpha\right),
$$

where the symbol $\mathcal{P}$ specifies the path-ordered exponential. All of the previous ingredients $S, a_{\Gamma}$ and $\mathbf{u}$ are channel-specific and their exact definition depends on whether one is examining the quark or gluon-initiated case. Their explicit expressions can be found in e.g. the appendix of ref. [32].

In all functions so far, we have highlighted the dependence on both the invariant mass $M$ of the $t \bar{t}$ pair and on the variable $\beta_{t}$. These are related by eq. (2.4) through the value of the top-quark mass $m_{t}$. In order to simplify the notation, from hereon we will drop the explicit $M$ dependence in the soft functions and in the evolution kernels, with the understanding that these objects still implicitly depend on $m_{t}$. 


\subsection{The soft function and its evolution}

To the best of our knowledge, the soft function for $t \bar{t}$ production which appears in eq. (2.6) has been defined for the first time in this work. In this section, we therefore compute the function at one-loop order, which is a necessary ingredient for resummation of the logarithms of $\mathcal{T}_{0}$ at NLL' accuracy and beyond.

\subsubsection{Calculation of the one-loop soft function}

The integrated soft functions in momentum space are given by

$$
\mathbf{S}_{B, i j}\left(\mathcal{T}_{s}, \beta_{t}, \theta, \mu\right)=\int \mathrm{d} k_{a}^{+} \mathrm{d} k_{b}^{+} \mathbf{S}_{i j}\left(k_{a}^{+}, k_{b}^{+}, \beta_{t}, \theta, \mu\right) \delta\left(\mathcal{T}_{s}-k_{b}^{+}-k_{a}^{+}\right),
$$

where the channel indices $i, j=\{q \bar{q}, \bar{q} q, g g\}$. The operatorial definition in SCET is given by eq. (A.30). We expand the soft functions in $\alpha_{s}$ as

$$
\mathbf{S}_{i j}\left(k_{a}^{+}, k_{b}^{+}, \beta_{t}, \theta, \mu\right)=\mathbf{s}_{i j}^{(0)} \delta\left(k_{a}^{+}\right) \delta\left(k_{b}^{+}\right)+\left(\frac{\alpha_{s}}{4 \pi}\right) \mathbf{S}_{i j}^{(1)}\left(k_{a}^{+}, k_{b}^{+}, \beta_{t}, \theta, \epsilon, \mu\right)+\mathcal{O}\left(\alpha_{\mathrm{s}}^{2}\right),
$$

where we have expressed the bare coupling $\alpha_{\mathrm{s}}^{0}$ in terms of the renormalised coupling $\alpha_{\mathrm{s}}(\mu)$ in the $\overline{\mathrm{MS}}$ scheme using the relation $Z_{\alpha_{s}} \alpha_{\mathrm{S}}(\mu) \mu^{2 \epsilon}=e^{-\gamma_{E} \epsilon}(4 \pi)^{\epsilon} \alpha_{\mathrm{S}}^{0}$. The leading order (LO) coefficients $\mathbf{s}_{i j}^{(0)}$ for the $q \bar{q}$ and $g g$ channels are defined in eq. (65) of ref. [32]. The next-to-leading order (NLO) bare soft functions in momentum space can be written as

$$
\mathbf{S}_{\mathrm{bare}, i j}^{(1)}\left(k_{a}^{+}, k_{b}^{+}, \beta_{t}, \theta, \epsilon, \mu\right)=\sum_{\alpha, \beta} \boldsymbol{w}_{i j}^{\alpha \beta} \hat{\mathcal{I}}_{\alpha \beta}\left(k_{a}^{+}, k_{b}^{+}, \beta_{t}, \theta, \epsilon, \mu\right),
$$

where the colour matrices $\boldsymbol{w}_{i j}^{\alpha \beta}$ for the $q \bar{q}$ and $g g$ channels are defined in eq. (71) of ref. [32] and the integrals are defined as

$$
\begin{aligned}
& \hat{\mathcal{I}}_{\alpha \beta}\left(k_{a}^{+},\right.\left.k_{b}^{+}, \beta_{t}, \theta, \epsilon, \mu\right)=-\frac{2\left(\mu^{2} e^{\gamma_{E}}\right)^{\epsilon}}{\pi^{1-\epsilon}} \int \mathrm{d}^{d} k \frac{v_{\alpha} \cdot v_{\beta}}{v_{\alpha} \cdot k v_{\beta} \cdot k} \delta\left(k^{2}\right) \Theta\left(k^{0}\right) \\
& \times\left[\delta\left(k_{a}^{+}-k \cdot n_{a}\right) \Theta\left(k \cdot n_{b}-k \cdot n_{a}\right) \delta\left(k_{b}^{+}\right)+\delta\left(k_{b}^{+}-k \cdot n_{b}\right) \Theta\left(k \cdot n_{a}-k \cdot n_{b}\right) \delta\left(k_{a}^{+}\right)\right] .
\end{aligned}
$$

The second line of the above equation represents the observable-specific measurement function; $v_{\alpha}, v_{\beta}=\left\{n_{a}, n_{b}, v_{3}, v_{4}\right\}$ are the velocities of the four external particles. Due to the particular structure of the measurement function at NLO, we may separate the integrals over the two hemispheres of the event, writing

$$
\hat{\mathcal{I}}_{\alpha \beta}\left(k_{a}^{+}, k_{b}^{+}, \beta_{t}, \theta, \epsilon, \mu\right)=\delta\left(k_{a}^{+}\right) \mathcal{I}_{\alpha \beta}\left(k_{b}^{+}, \beta_{t}, \theta, \epsilon, \mu\right)+\delta\left(k_{b}^{+}\right) \mathcal{I}_{\alpha \beta}\left(k_{a}^{+}, \beta_{t}, \theta, \epsilon, \mu\right),
$$

where the integrals $\mathcal{I}_{\alpha \beta}$ refer to the contribution of a single hemisphere.

Analogous to the decomposition for the renormalised function, it follows from eqs. (2.15) and (2.17) that

$$
\begin{aligned}
\mathbf{S}_{B, \text { bare }, i j}^{(1)}\left(\mathcal{T}_{s}, \beta_{t}, \theta, \mu\right) & =\int \mathrm{d} k_{a}^{+} \mathrm{d} k_{b}^{+} \mathbf{S}_{\mathrm{bare}, i j}^{(1)}\left(k_{a}^{+}, k_{b}^{+}, \beta_{t}, \theta, \epsilon, \mu\right) \delta\left(\mathcal{T}_{s}-k_{a}^{+}-k_{b}^{+}\right) \\
& =2 \sum_{\alpha, \beta} \boldsymbol{w}_{i j}^{\alpha \beta}\left(\int \mathrm{d} l^{+} \mathcal{I}_{\alpha \beta}\left(l^{+}, \beta_{t}, \theta, \epsilon, \mu\right) \delta\left(\mathcal{T}_{s}-l^{+}\right)\right) .
\end{aligned}
$$


In the following, we therefore focus on the calculation of the single hemisphere integrals $\mathcal{I}_{\alpha \beta}\left(l^{+}, \beta_{t}, \theta, \epsilon, \mu\right)$ which we normalise as

$$
\mathcal{I}_{\alpha \beta}\left(l^{+}, \beta_{t}, \theta, \epsilon, \mu\right)=-\frac{2\left(\mu^{2} e^{\gamma_{E}}\right)^{\epsilon}}{\pi^{1-\epsilon}} I_{\alpha \beta}\left(l^{+}, \beta_{t}, \theta, \epsilon, \mu\right) .
$$

The symmetries of the integrals mean that we have the following relations:

$$
\begin{aligned}
I_{v_{4} v_{4}} & =I_{v_{3} v_{3}}(\theta \rightarrow \pi-\theta) \\
I_{n_{a} v_{4}} & =I_{n_{a} v_{3}}(\theta \rightarrow \pi-\theta) \\
I_{n_{b} v_{4}} & =I_{n_{b} v_{3}}(\theta \rightarrow \pi-\theta)
\end{aligned}
$$

The integrals which feature only light-like legs are also needed in the computation of the 0-jettiness soft function for colour singlet production, and as such appear in e.g. refs. $[67,68,70]$. In appendix $\mathrm{B}$, we evaluate analytically the pole structure of all remaining required integrals $I_{\alpha \beta}$ at this order. We were unable to obtain closed analytic expressions for the finite parts, however, and have therefore evaluated these onefold integrals numerically.

Thus far we have discussed the computation of the bare soft function at $\mathcal{O}\left(\alpha_{\mathrm{s}}\right)$. This object still contains ultraviolet poles in $\epsilon$ and must therefore be renormalised. In Laplace space, the renormalisation can be performed by multiplying both sides of the bare soft function by a matrix-valued renormalisation factor

$$
\tilde{\mathbf{S}}_{B, i j}\left(L, \beta_{t}, \theta, \mu\right)=\mathbf{Z}_{S, i j}^{\dagger}\left(\alpha_{\mathrm{s}}, L, \epsilon\right) \tilde{\mathbf{S}}_{B, \text { bare }, i j}\left(L, \beta_{t}, \theta\right) \mathbf{Z}_{S, i j}\left(\alpha_{\mathrm{s}}, L, \epsilon\right),
$$

where we have defined $L=\ln \left(\kappa^{2} / \mu^{2}\right)$. At one-loop order, the effect of eq. (2.23) is simply to remove the UV poles from $\tilde{\mathbf{S}}_{B \text {,bare }}^{(1)}$. The renormalised soft function at this order is therefore obtained by taking the finite part of the bare function. Since the bare soft function in eq. (2.23) is independent of $\mu$, both the renormalised function and the renormalisation factor obey a renormalisation group equation, which we shall discuss in the following section.

The renormalisation procedure also completely determines the structure of the $\mathcal{O}\left(\alpha_{\mathrm{s}}\right)$ term $\mathbf{Z}_{S}^{(1)}$, which allows us to extract the soft anomalous dimension at one-loop. We verified that by doing so, this object satisfies consistency relations required by RG invariance of eq. (2.6) (see eq. (2.35)). In addition, by exploiting this relation at one order higher, we are able to extract the soft anomalous dimension at two-loop order.

\subsubsection{Solving the soft RG equations at fixed order}

A resummation at full NNLL' accuracy would require knowledge of the two-loop contributions to the soft function, which have not yet been calculated. It is, however, possible to obtain partial knowledge about the two-loop function by solving the renormalisation group evolution equations at fixed order. In this way, one can obtain the logarithmic terms at $\mathcal{O}\left(\alpha_{s}^{2}\right)$ expressed in terms of coefficients at lower order, leaving only the term proportional to $\delta\left(\mathcal{T}_{0}\right)$ to be determined by an explicit calculation.

The soft functions in Laplace space satisfy the following renormalisation group equations

$$
\frac{\mathrm{d}}{\mathrm{d} \ln \mu} \tilde{\mathbf{S}}_{B}\left(L, \beta_{t}, \theta, \mu\right)=\left[\Gamma_{\text {cusp }} L-\gamma^{s^{\dagger}}\right] \tilde{\mathbf{S}}_{B}\left(L, \beta_{t}, \theta, \mu\right)+\tilde{\mathbf{S}}_{B}\left(L, \beta_{t}, \theta, \mu\right)\left[\Gamma_{\text {cusp }} L-\gamma^{s}\right],
$$


where we have dropped the channel subscript for simplicity. Since the expansions of $\Gamma_{\text {cusp }}$ and the non-cusp soft anomalous dimension matrices $\gamma^{s}$ start at $\mathcal{O}\left(\alpha_{\mathrm{s}}\right)$, defining

$$
\tilde{\mathbf{S}}_{B}\left(L, \beta_{t}, \theta, \mu\right)=\mathbf{s}^{(0)}+\frac{\alpha_{s}}{4 \pi} \tilde{\mathbf{S}}_{B}^{(1)}+\left(\frac{\alpha_{s}}{4 \pi}\right)^{2} \tilde{\mathbf{S}}_{B}^{(2)}+\mathcal{O}\left(\alpha_{s}^{3}\right)
$$

and expanding eq. (2.24) at NNLO we have

$$
\frac{\mathrm{d}}{\mathrm{d} L} \tilde{\mathbf{S}}_{B}^{(2)}=\frac{1}{2} \tilde{\mathbf{S}}_{B}^{(1)}\left[\left(-\Gamma_{\text {cusp }}^{(0)} L-\beta_{0}\right)+\boldsymbol{\gamma}^{s(0)}\right]+\frac{1}{2} \mathbf{s}^{(0)}\left[-\Gamma_{\text {cusp }}^{(1)} L+\gamma^{s(1)}\right]+\text { h.c. }
$$

Denoting further the logarithmic coefficients of the soft function as

$$
\tilde{\mathbf{S}}_{B}\left(L, \beta_{t}, \theta, \mu\right)=\sum_{n=0}^{\infty} \sum_{m=0}^{2 n}\left(\frac{\alpha_{\mathrm{s}}}{4 \pi}\right)^{n} \tilde{\mathbf{S}}_{B}^{(n, m)}\left(\beta_{t}, \theta\right) L^{m}
$$

and again suppressing arguments for brevity, we find the solution

$$
\begin{aligned}
& \tilde{\mathbf{S}}_{B}^{(2,4)}=-\frac{1}{8} \tilde{\mathbf{S}}_{B}^{(1,2)} \Gamma_{\text {cusp }}^{(0)}+\text { h.c. } \\
& \tilde{\mathbf{S}}_{B}^{(2,3)}=\frac{1}{6}\left(-\tilde{\mathbf{S}}_{B}^{(1,1)} \Gamma_{\text {cusp }}^{(0)}+\tilde{\mathbf{S}}_{B}^{(1,2)} \boldsymbol{\gamma}^{s(0)}-\beta_{0} \tilde{\mathbf{S}}_{B}^{(1,2)}\right)+\text { h.c. } \\
& \tilde{\mathbf{S}}_{B}^{(2,2)}=\frac{1}{4}\left(-\tilde{\mathbf{S}}_{B}^{(1,0)} \Gamma_{\text {cusp }}^{(0)}+\tilde{\mathbf{S}}_{B}^{(1,1)} \boldsymbol{\gamma}^{s(0)}-\mathbf{s}^{(0)} \Gamma_{\text {cusp }}^{(1)}-\beta_{0} \tilde{\mathbf{S}}_{B}^{(1,1)}\right)+\text { h.c. } \\
& \tilde{\mathbf{S}}_{B}^{(2,1)}=\frac{1}{2}\left(\tilde{\mathbf{S}}_{B}^{(1,0)} \boldsymbol{\gamma}^{s(0)}+\mathbf{s}^{(0)} \boldsymbol{\gamma}^{s(1)}-\beta_{0} \tilde{\mathbf{S}}_{B}^{(1,0)}\right)+\text { h.c. }
\end{aligned}
$$

Upon transforming back to momentum space, we thus have all the soft ingredients necessary to construct the $\mathcal{T}_{0}$ spectrum at approximate NNLO. We are only missing the term $\tilde{\mathbf{S}}_{B}^{(2,0)}$, which contributes only at the point $\mathcal{T}_{0}=0$ and must be computed separately. This means that once we combine these with the contributions coming from the beam and hard functions we are able to cancel all the singular pieces at small $\mathcal{T}_{0}$ of the NLO calculation for $t \bar{t}+$ jet production.

\subsubsection{Evolution}

In Laplace space, the all-order solutions of the soft RG evolution in eq. (2.24) can be written as

$$
\tilde{\mathbf{S}}_{B}\left(L, \beta_{t}, \theta, \mu\right)=\mathbf{V}^{\dagger}\left(\kappa, \beta_{t}, \theta, \mu_{s}, \mu\right) \tilde{\mathbf{S}}_{B}\left(L, \beta_{t}, \theta, \mu_{s}\right) \mathbf{V}\left(\kappa, \beta_{t}, \theta, \mu_{s}, \mu\right),
$$

where the unitary matrix $\mathbf{V}$ satisfies the differential equation

$$
\frac{\mathrm{d}}{\mathrm{d} \ln \mu} \mathbf{V}\left(\kappa, \beta_{t}, \theta, \mu_{s}, \mu\right)=\left(\Gamma_{\text {cusp }} \ln \frac{\kappa^{2}}{\mu^{2}}-\gamma_{s}\right) \mathbf{V}\left(\kappa, \beta_{t}, \theta, \mu_{s}, \mu\right)
$$

and the soft scale $\mu_{s} \sim \mathcal{T}_{0}$ minimises the logarithms in the soft functions. Proceeding analogously to the hard function case and resumming the soft logarithms while evolving from the soft scale to a generic scale $\mu$, we find the solution

$$
\mathbf{V}\left(\kappa, \beta_{t}, \theta, \mu_{s}, \mu\right)=\exp \left[2 S\left(\mu_{s}, \mu\right)\right]\left(\frac{\kappa^{2}}{\mu_{s}^{2}}\right)^{-a_{\Gamma}\left(\mu_{s}, \mu\right)} \mathbf{v}\left(\beta_{t}, \theta, \mu_{s}, \mu\right),
$$


with the non-cusp soft evolution matrices given by

$$
\mathbf{v}\left(\beta_{t}, \theta, \mu_{s}, \mu\right)=\mathcal{P} \exp \left\{-\int_{\alpha_{s}\left(\mu_{s}\right)}^{\alpha_{s}(\mu)} \frac{\mathrm{d} \alpha}{\beta(\alpha)} \gamma^{s}\left(\beta_{t}, \theta, \alpha\right)\right\} .
$$

Substituting these ingredients into eq. (2.29) we obtain

$$
\tilde{\mathbf{S}}_{B}\left(L, \beta_{t}, \theta, \mu\right)=\exp \left[4 S\left(\mu_{s}, \mu\right)\right] \mathbf{v}^{\dagger}\left(\beta_{t}, \theta, \mu_{s}, \mu\right) \tilde{\mathbf{S}}_{B}\left(\partial_{\eta_{s}}, \beta_{t}, \theta, \mu_{s}\right) \mathbf{v}\left(\beta_{t}, \theta, \mu_{s}, \mu\right)\left(\frac{\kappa^{2}}{\mu_{s}^{2}}\right)^{\eta_{s}},
$$

where after taking the derivatives we set $\eta_{s} \equiv-2 a_{\Gamma}\left(\mu_{s}, \mu\right)$. In the last equation we have rewritten the logarithms appearing as an argument of the soft function in terms of partial derivatives acting on the last factor [71, 72]. Transforming back to momentum space yields

$$
\begin{aligned}
\mathbf{S}_{B}\left(l^{+}, \beta_{t}, \theta, \mu\right)= & \exp \left[4 S\left(\mu_{s}, \mu\right)\right] \mathbf{v}^{\dagger}\left(\beta_{t}, \theta, \mu_{s}, \mu\right) \tilde{\mathbf{S}}_{B}\left(\partial_{\eta_{s}}, \beta_{t}, \theta, \mu_{s}\right) \mathbf{v}\left(\beta_{t}, \theta, \mu_{s}, \mu\right) \\
& \times \frac{1}{l^{+}}\left(\frac{l^{+}}{\mu_{s}}\right)^{2 \eta_{s}} \frac{e^{-2 \gamma_{E} \eta_{s}}}{\Gamma\left(2 \eta_{s}\right)} .
\end{aligned}
$$

Due to the RG invariance of the full cross section we have the following relation between the non-cusp anomalous dimensions of the hard, soft, and beam functions

$$
\gamma^{s}=\gamma^{h}+\gamma^{B} \mathbf{1}
$$

where the non-diagonal part of the soft anomalous dimension arises entirely from the non-cusp anomalous dimension of the hard function and $\gamma^{B}$ is the non-cusp anomalous dimension for the beam function. The evolution formula in eq. (2.34) for the soft function can therefore be rewritten as

$$
\begin{aligned}
\mathbf{S}_{B}\left(l^{+}, \beta_{t}, \theta, \mu\right)= & \exp \left[4 S\left(\mu_{s}, \mu\right)+2 a_{\gamma^{B}}\left(\mu_{s}, \mu\right)\right] \\
& \times \mathbf{u}^{\dagger}\left(\beta_{t}, \theta, \mu, \mu_{s}\right) \tilde{\mathbf{S}}_{B}\left(\partial_{\eta_{s}}, \beta_{t}, \theta, \mu_{s}\right) \mathbf{u}\left(\beta_{t}, \theta, \mu, \mu_{s}\right) \frac{1}{l^{+}}\left(\frac{l^{+}}{\mu_{s}}\right)^{2 \eta_{s}} \frac{e^{-2 \gamma_{E} \eta_{s}}}{\Gamma\left(2 \eta_{s}\right)},
\end{aligned}
$$

where the order of the scale arguments in the $\mathbf{u}$ evolution matrices is now inverted relative to the $\mathbf{v}$ matrices and

$$
a_{\gamma^{B}}\left(\mu_{s}, \mu\right)=-\int_{\alpha_{s}\left(\mu_{s}\right)}^{\alpha_{s}(\mu)} \mathrm{d} \alpha \frac{\gamma^{B}(\alpha)}{\beta(\alpha)}
$$

\subsection{The beam functions and their evolution}

The process-independent $\mathcal{T}_{0}$ beam functions $B_{i}$ have been computed up to $\mathrm{N}^{3} \mathrm{LO}$ accuracy and are available in the literature [62-66]. The quark and gluon beam functions satisfy the following $\mathrm{RG}$ equation in Laplace space

$$
\frac{\mathrm{d}}{\mathrm{d} \ln \mu} \tilde{B}_{i}\left(L_{c}, z, \mu\right)=\left[-2 \Gamma_{\text {cusp }}\left(\alpha_{s}\right) L_{c}+\gamma_{i}^{B}\left(\alpha_{s}\right)\right] \tilde{B}_{i}\left(L_{c}, z, \mu\right),
$$


where the index $i=\{q, \bar{q}, g\}, L_{c}=\ln \left[(M \kappa) / \mu^{2}\right]$ and $\Gamma_{\text {cusp }}=C_{D} \gamma_{\text {cusp }}$ with $C_{D}=\left\{C_{F}, C_{A}\right\}$ for the quark and the gluon beam functions respectively. The explicit expressions for the non-cusp beam anomalous dimensions $\gamma_{i}^{B}$ up to NNLO can be found in e.g. appendix D of ref. [57]. Dropping the flavour index for brevity, the evolution equation has the solution

$$
\tilde{B}\left(L_{c}, z, \mu\right)=\exp \left[-4 S\left(\mu_{B}, \mu\right)-a_{\gamma^{B}}\left(\mu_{B}, \mu\right)\right] \tilde{B}\left(\partial_{\eta_{B}}, z, \mu_{B}\right)\left(\frac{M \kappa}{\mu_{B}^{2}}\right)^{\eta_{B}}
$$

where after taking the derivative we set $\eta_{B} \equiv 2 a_{\Gamma}\left(\mu_{B}, \mu\right)$ and $\mu_{B} \sim \sqrt{\mathcal{T}_{0} M}$ is the beam scale. Taking the inverse transform again we find that, in momentum space,

$$
B(t, z, \mu)=\exp \left[-4 S\left(\mu_{B}, \mu\right)-a_{\gamma^{B}}\left(\mu_{B}, \mu\right)\right] \tilde{B}\left(\partial_{\eta_{B}}, z, \mu_{B}\right) \frac{1}{t}\left(\frac{t}{\mu_{B}^{2}}\right)^{\eta_{B}} \frac{e^{-\gamma_{E} \eta_{B}}}{\Gamma\left(\eta_{B}\right)} .
$$

\section{Resummation via renormalisation group evolution}

In this section, we combine the factorisation theorem and the perturbative ingredients presented in section 2 to resum logarithms of $\mathcal{T}_{0} / M$. We present explicit formulæ for the resummed $\mathcal{T}_{0}$ spectrum at NLL', NNLL and NNLL' order.

\subsection{All-order solutions of the RG equations}

Substituting the resummed expressions for the ingredients of eq. (2.6) which we have presented in section 2 and after integrating over the virtualities $t_{a}$ and $t_{b}$, we are able to write the resummed cross section in a compact form as

$$
\begin{aligned}
\frac{\mathrm{d} \sigma}{\mathrm{d} \Phi_{0} \mathrm{~d} \tau_{B}}= & U\left(\mu_{h}, \mu_{B}, \mu_{s}, L_{h}, L_{s}\right) \\
& \times \operatorname{Tr}\left\{\mathbf{u}\left(\beta_{t}, \theta, \mu_{h}, \mu_{s}\right) \mathbf{H}\left(M, \beta_{t}, \theta, \mu_{h}\right) \mathbf{u}^{\dagger}\left(\beta_{t}, \theta, \mu_{h}, \mu_{s}\right) \tilde{\mathbf{S}}_{B}\left(\partial_{\eta_{s}}+L_{s}, \beta_{t}, \theta, \mu_{s}\right)\right\} \\
& \times \tilde{B}_{a}\left(\partial_{\eta_{B}}+L_{B}, z_{a}, \mu_{B}\right) \tilde{B}_{b}\left(\partial_{\eta_{B}^{\prime}}+L_{B}, z_{b}, \mu_{B}\right) \frac{1}{\tau_{B}^{1-\eta_{\text {tot }}}} \frac{e^{-\gamma_{E} \eta_{\text {tot }}}}{\Gamma\left(\eta_{\text {tot }}\right)}
\end{aligned}
$$

The derivative terms inside the arguments of the soft and beam functions act on the factor in the last line of the previous equation, which we refer to as the generating function. We have also introduced the quantity $\eta_{\text {tot }}=2 \eta_{s}+\eta_{B}+\eta_{B}^{\prime}$. We recall that after taking the derivatives one has to set $\eta_{B}^{\prime}=\eta_{B}=2 a_{\Gamma}\left(\mu_{B}, \mu\right)$ and $\eta_{s} \equiv-2 a_{\Gamma}\left(\mu_{s}, \mu\right)$. In the previous formula we have collected some of the color diagonal evolution terms in the common factor

$$
\begin{aligned}
& U\left(\mu_{h}, \mu_{B}, \mu_{s}, L_{h}, L_{s}\right)= \\
& \quad \exp \left[4 S\left(\mu_{h}, \mu_{B}\right)+4 S\left(\mu_{s}, \mu_{B}\right)+2 a_{\gamma^{B}}\left(\mu_{s}, \mu_{B}\right)-2 a_{\Gamma}\left(\mu_{h}, \mu_{B}\right) L_{h}-2 a_{\Gamma}\left(\mu_{s}, \mu_{B}\right) L_{s}\right] .
\end{aligned}
$$




\begin{tabular}{|c|cccc|}
\hline Accuracy & b.c. & $\beta, \Gamma_{\text {cusp }}$ & $\gamma^{x}$ & n.s. matching \\
\hline NLL $^{\prime}$ & $\mathcal{O}\left(\alpha_{\mathrm{s}}\right)$ & 2-loop & 1-loop & $\mathrm{LO}_{1}$ \\
NNLL & $\mathcal{O}\left(\alpha_{\mathrm{s}}\right)$ & 3-loop & 2-loop & $\mathrm{LO}_{1}$ \\
NNLL $^{\prime}$ & $\mathcal{O}\left(\alpha_{\mathrm{s}}^{2}\right)$ & 3-loop & 2-loop & $\mathrm{NLO}_{1}$ \\
\hline
\end{tabular}

Table 1. Definition of resummed accuracies in terms of the required perturbative order of the resummation ingredients and of the boundary conditions. The appropriate fixed order matching is also indicated.

We explicitly write the beam, soft and hard logarithms as $L_{B}=\ln \left(M^{2} / \mu_{B}^{2}\right), L_{s}=\ln \left(M^{2} / \mu_{s}^{2}\right)$ and $L_{h}=\ln \left(M^{2} / \mu_{h}^{2}\right)$. For the derivation of the formula above we have used the relations

$$
\begin{aligned}
\mathbf{u}\left(\beta_{t}, \theta, \mu_{c}, \mu_{a}\right) \mathbf{u}\left(\beta_{t}, \theta, \mu_{b}, \mu_{c}\right) & =\mathbf{u}\left(\beta_{t}, \theta, \mu_{b}, \mu_{a}\right), \\
a_{\Gamma}\left(\mu_{a}, \mu_{c}\right) & =a_{\Gamma}\left(\mu_{a}, \mu_{b}\right)+a_{\Gamma}\left(\mu_{b}, \mu_{c}\right), \\
a_{\gamma^{i}}\left(\mu_{a}, \mu_{c}\right) & =a_{\gamma^{i}}\left(\mu_{a}, \mu_{b}\right)+a_{\gamma^{i}}\left(\mu_{b}, \mu_{c}\right), \\
S\left(\mu_{a}, \mu_{b}\right)-S\left(\mu_{c}, \mu_{b}\right) & =S\left(\mu_{a}, \mu_{c}\right)-a_{\Gamma}\left(\mu_{c}, \mu_{b}\right) \ln \frac{\mu_{a}}{\mu_{c}},
\end{aligned}
$$

to simplify the final expressions.

The expression in eq. (3.1) is our master formula and the primary outcome of this work. It is formally valid at all logarithmic orders. It is possible to evaluate it at NLL', NNLL and NNLL' depending on the order in $\alpha_{s}$ at which the anomalous dimensions and the boundary terms are available.

In order to evaluate $\mathbf{u}$ we first find the matrix $\boldsymbol{\Lambda}$ which diagonalises the LO non-cusp hard anomalous dimension

$$
\gamma_{D}^{h(0)}=\Lambda^{-1} \gamma^{h(0)} \boldsymbol{\Lambda}
$$

and define the vector $\vec{\gamma}^{h(0)}$ consisting of the eigenvalues of the diagonal matrix $\gamma_{D}^{h(0)}$. The solution of the non-cusp evolution matrix in eq. (2.14) up to NNLL can then be obtained perturbatively as an expansion in $\alpha_{s}$ following appendix $\mathrm{A}$ of ref. [32] and the references therein $[73,74]$. We find

$$
\mathbf{u}^{\mathrm{NNLL}}\left(\beta_{t}, \theta, \mu_{h}, \mu\right)=\left[\boldsymbol{\Lambda}\left(1+\frac{\alpha_{s}(\mu)}{4 \pi} \boldsymbol{K}\right)\left(\left[\frac{\alpha_{s}\left(\mu_{h}\right)}{\alpha_{s}(\mu)}\right]^{\frac{\vec{\gamma}^{h(0)}}{2 \beta_{0}}}\right)_{D}\left(1-\frac{\alpha_{s}\left(\mu_{h}\right)}{4 \pi} \boldsymbol{K}\right) \boldsymbol{\Lambda}^{-1}\right]_{\mathcal{O}\left(\alpha_{\mathrm{s}}\right)}
$$

where $[\ldots]_{\mathcal{O}\left(\alpha_{\mathrm{s}}\right)}$ indicates the expansion up to the first power of $\alpha_{\mathrm{s}}$ and the elements of the matrix $\boldsymbol{K}$ are given by

$$
K_{i j}=\delta_{i j} \vec{\gamma}_{i}^{h(0)} \frac{\beta_{1}}{2 \beta_{0}^{2}}-\frac{\left[\boldsymbol{\Lambda}^{-1} \gamma^{h(1)} \boldsymbol{\Lambda}\right]_{i j}}{2 \beta_{0}+\vec{\gamma}_{i}^{h(0)}-\vec{\gamma}_{j}^{h(0)}}
$$

For later convenience we separate the contributions of the NLL evolution from the pure NNLL corrections which enter at $\mathcal{O}\left(\alpha_{s}\right)$ in the logarithmic counting. In practice we define 
the quantities

$$
\begin{aligned}
& \mathbf{u}^{\mathrm{NLL}}\left(\beta_{t}, \theta, \mu_{h}, \mu\right)=\boldsymbol{\Lambda}\left(\left[\frac{\alpha_{s}\left(\mu_{h}\right)}{\alpha_{s}(\mu)}\right]^{\frac{\vec{\gamma}^{h(0)}}{2 \beta_{0}}}\right)_{D} \boldsymbol{\Lambda}^{-1} \text { and }
\end{aligned}
$$

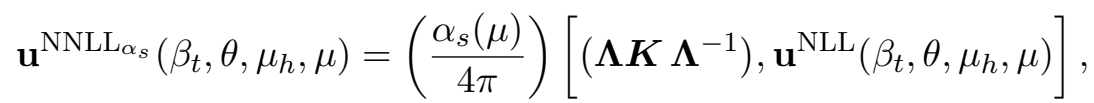

(where $[x, y]$ denotes the usual commutator) such that the NNLL non-cusp evolution matrix is written as

$$
\mathbf{u}^{\mathrm{NNLL}}\left(\beta_{t}, \theta, \mu_{h}, \mu\right)=\mathbf{u}^{\mathrm{NLL}}\left(\beta_{t}, \theta, \mu_{h}, \mu\right)+\mathbf{u}^{\mathrm{NNLL}_{\alpha_{s}}}\left(\beta_{t}, \theta, \mu_{h}, \mu\right) .
$$

\section{2 $\quad \mathrm{NLL}^{\prime}$ and NNLL formulæ}

Beginning from the general resummation formula in eq. (3.1), it is possible to write the formula one would obtain at a given resummed accuracy explicitly. To this end, we use the expansion of the soft function defined in eq. (2.25) and define the following perturbative coefficients for the hard and beam functions

$$
\begin{aligned}
\mathbf{H}\left(M, \beta_{t}, \theta, \mu_{h}\right) & =\frac{\alpha_{s}^{2}}{d_{R}}\left[\mathbf{H}^{(0)}+\frac{\alpha_{s}}{4 \pi} \mathbf{H}^{(1)}+\left(\frac{\alpha_{s}}{4 \pi}\right)^{2} \mathbf{H}^{(2)}+\mathcal{O}\left(\alpha_{s}^{3}\right)\right], \\
\tilde{B}_{i}\left(L_{c}, x, \mu_{B}\right) & =\tilde{B}_{i}^{(0)}+\frac{\alpha_{s}}{4 \pi} \tilde{B}_{i}^{(1)}+\left(\frac{\alpha_{s}}{4 \pi}\right)^{2} \tilde{B}_{i}^{(2)}+\mathcal{O}\left(\alpha_{s}^{3}\right),
\end{aligned}
$$

with $d_{R}$ being the dimension of the quark or the gluon $\mathrm{SU}\left(N_{c}\right)$ colour representation such that $d_{R}=\left\{N_{c}, N_{c}^{2}-1\right\}$ for the $q \bar{q}$ and $g g$ channels respectively. In table 1 we summarise the perturbative order to which the anomalous dimensions and beta function must be evaluated in order to reach a given accuracy.

The explicit expression at NLL' then reads

$$
\begin{aligned}
\frac{\mathrm{d} \sigma^{\mathrm{NLL}}{ }^{\prime}}{\mathrm{d} \Phi_{0} \mathrm{~d} \tau_{B}}= & U^{\mathrm{NLL}}\left(\mu_{h}, \mu_{B}, \mu_{s}, L_{h}, L_{s}\right) \\
& \times\left\{\left[\operatorname{Tr}\left\{\mathbf{u}^{\mathrm{NLL}}\left(\beta_{t}, \theta, \mu_{h}, \mu_{s}\right) \mathbf{H}^{(0)}\left(M, \beta_{t}, \theta, \mu_{h}\right) \mathbf{u}^{\dagger \mathrm{NLL}}\left(\beta_{t}, \theta, \mu_{h}, \mu_{s}\right) \mathbf{s}^{(0)}\right\}\right.\right. \\
& +\frac{\alpha_{s}\left(\mu_{h}\right)}{4 \pi} \operatorname{Tr}\left\{\mathbf{u}^{\mathrm{NLL}}\left(\beta_{t}, \theta, \mu_{h}, \mu_{s}\right) \mathbf{H}^{(1)}\left(M, \beta_{t}, \theta, \mu_{h}\right) \mathbf{u}^{\dagger \mathrm{NLL}}\left(\beta_{t}, \theta, \mu_{h}, \mu_{s}\right) \mathbf{s}^{(0)}\right\} \\
& +\frac{\alpha_{s}\left(\mu_{s}\right)}{4 \pi} \operatorname{Tr}\left\{\mathbf{u}^{\mathrm{NLL}}\left(\beta_{t}, \theta, \mu_{h}, \mu_{s}\right) \mathbf{H}^{(0)}\left(M, \beta_{t}, \theta, \mu_{h}\right) \mathbf{u}^{\dagger \mathrm{NLL}}\left(\beta_{t}, \theta, \mu_{h}, \mu_{s}\right)\right. \\
& \left.\left.\times \tilde{\mathbf{S}}_{B}^{(1)}\left(\partial_{\eta_{s}}+L_{s}, \beta_{t}, \theta, \mu_{s}\right)\right\}\right] \times \tilde{B}_{a}^{(0)}\left(z_{a}, \mu_{B}\right) \tilde{B}_{b}^{(0)}\left(z_{b}, \mu_{B}\right) \\
& +\frac{\alpha_{s}\left(\mu_{B}\right)}{4 \pi} \operatorname{Tr}\left\{\mathbf{u}^{\mathrm{NLL}}\left(\beta_{t}, \theta, \mu_{h}, \mu_{s}\right) \mathbf{H}^{(0)}\left(M, \beta_{t}, \theta, \mu_{h}\right) \mathbf{u}^{\dagger \mathrm{NLL}}\left(\beta_{t}, \theta, \mu_{h}, \mu_{s}\right) \mathbf{s}^{(0)}\right\} \\
& \left.\times\left(\tilde{B}_{a}^{(1)}\left(\partial_{\eta_{B}}+L_{B}, z_{a}, \mu_{B}\right) \tilde{B}_{b}^{(0)}\left(z_{b}, \mu_{B}\right)+\tilde{B}_{a}^{(0)}\left(z_{a}, \mu_{B}\right) \tilde{B}_{b}^{(1)}\left(\partial_{\eta_{B}^{\prime}}+L_{B}, z_{b}, \mu_{B}\right)\right)\right\} \\
& \times \frac{1}{\tau_{B}^{1-\eta_{\mathrm{tot}}^{\mathrm{NLL}}} \frac{e^{-\gamma_{E}} \eta_{\mathrm{tot}}^{\mathrm{NLL}}}{\Gamma\left(\eta_{\mathrm{tot}}^{\mathrm{NLL}}\right)},}
\end{aligned}
$$


where the factor $U^{\mathrm{NLL}}$ is given by eq. (3.2) with the expressions for $S, a_{\Gamma}$ and $a_{\gamma^{B}}$ expanded only up to NLL accuracy.

We now consider accuracies beyond NLL'. We write the additional $\mathcal{O}\left(\alpha_{s}\right)$ correction that must be added to the NLL' result in eq. (3.12) to reach NNLL accuracy as

$$
\frac{\mathrm{d} \sigma^{\mathrm{NNLL}}}{\mathrm{d} \Phi_{0} \mathrm{~d} \tau_{B}}=\frac{\mathrm{d} \sigma^{\mathrm{NLL}}}{\mathrm{d} \Phi_{0} \mathrm{~d} \tau_{B}}+\frac{\mathrm{d} \sigma^{\mathrm{NNLL}} \alpha_{\alpha_{s}}}{\mathrm{~d} \Phi_{0} \mathrm{~d} \tau_{B}}
$$

Given that the non-diagonal evolution term in eq. (3.5) is only known perturbatively, at NNLL we also need to expand the exponential prefactor $U$ which performs the diagonal evolution to order $\alpha_{s}$ to maintain consistency with the perturbative expansion of the non-diagonal evolution term. ${ }^{6}$

The $\mathcal{O}\left(\alpha_{s}\right)$ contribution that ensures NNLL accuracy is then given by

$$
\begin{aligned}
& \frac{\mathrm{d} \sigma^{\mathrm{NNLL}_{\alpha_{s}}}}{\mathrm{~d} \Phi_{0} \mathrm{~d} \tau_{B}}=U^{\mathrm{NLL}}\left(\mu_{h}, \mu_{B}, \mu_{s}, L_{h}, L_{s}\right) \frac{\eta_{\mathrm{tot}}^{\mathrm{NLL}}}{\tau_{B}^{1-\eta_{\mathrm{tot}}^{\mathrm{NLL}}}} \frac{e^{-\gamma_{E}} \eta_{\mathrm{tot}}^{\mathrm{NLL}}}{\Gamma\left(1+\eta_{\mathrm{tot}}^{\mathrm{NLL}}\right)} \\
& \times\left\{\left[\left(4 S^{\left(\alpha_{s}\right)}\left(\mu_{h}, \mu_{B}\right)+4 S^{\left(\alpha_{s}\right)}\left(\mu_{s}, \mu_{B}\right)+2 a_{\gamma^{B}}^{\left(\alpha_{s}\right)}\left(\mu_{s}, \mu_{B}\right)\right.\right.\right. \\
& \left.-2 a_{\Gamma}^{\left(\alpha_{s}^{2}\right)}\left(\mu_{h}, \mu_{B}\right) L_{h}-2 a_{\Gamma}^{\left(\alpha_{s}^{2}\right)}\left(\mu_{s}, \mu_{B}\right) L_{s}+\eta_{\mathrm{tot}}^{\left(\alpha_{s}^{2}\right)}\left(\ln \left(\tau_{B}\right)-\gamma_{E}-\psi_{0}\left(\eta_{\mathrm{tot}}^{\mathrm{NLL}}\right)\right)\right) \\
& \left.\times \operatorname{Tr}\left\{\mathbf{u}^{\mathrm{NLL}}\left(\beta_{t}, \theta, \mu_{h}, \mu_{s}\right) \mathbf{H}^{(0)}\left(M, \beta_{t}, \theta, \mu_{h}\right) \mathbf{u}^{\dagger \mathrm{NLL}}\left(\beta_{t}, \theta, \mu_{h}, \mu_{s}\right) \mathbf{s}^{(0)}\right\}\right] \\
& +\operatorname{Tr}\left\{\mathbf{u}^{\mathrm{NNLL}_{\alpha_{s}}}\left(\beta_{t}, \theta, \mu_{h}, \mu_{s}\right) \mathbf{H}^{(0)}\left(M, \beta_{t}, \theta, \mu_{h}\right) \mathbf{u}^{\dagger N L L}\left(\beta_{t}, \theta, \mu_{h}, \mu_{s}\right) \mathbf{s}^{(0)}\right\} \\
& \left.+\operatorname{Tr}\left\{\mathbf{u}^{\mathrm{NLL}}\left(\beta_{t}, \theta, \mu_{h}, \mu_{s}\right) \mathbf{H}^{(0)}\left(M, \beta_{t}, \theta, \mu_{h}\right) \mathbf{u}^{\dagger \mathrm{NNLL}_{\alpha_{s}}}\left(\beta_{t}, \theta, \mu_{h}, \mu_{s}\right) \mathbf{s}^{(0)}\right\}\right\} \\
& \times \tilde{B}_{a}^{(0)}\left(z_{a}, \mu_{B}\right) \tilde{B}_{b}^{(0)}\left(z_{b}, \mu_{B}\right) .
\end{aligned}
$$

In the equation above the superscripts on the $S, a_{\gamma^{B}}$ and $a_{\Gamma}$ functions indicate the correction at a specific order in $\alpha_{s}{ }^{7}$ We have also consistently expanded the generating function at the appropriate order. Since after the expansion no derivatives appear in the arguments of the soft and beam functions, we are free to move the generating function to the first line.

An alternative to eq. (3.14), which formally still gives the same NNLL accuracy, involves upgrading its first line to use the NNLL formulæ for the diagonal evolution factor $U$ and for the generating function. One must then drop the expansion appearing inside the parentheses in the second and third line. We will discuss the numerical impact of this difference in section 4 .

\footnotetext{
${ }^{6}$ We are always considering the logarithmic counting where $\alpha_{s} L \sim 1$. Note that for the $a_{\Gamma}$ contributions the expansion must be performed to one order higher.

${ }^{7}$ Note that, for simplicity, we have not factored out any couplings from these terms. To give a specific example, $S^{\left(\alpha_{\mathrm{s}}\right)}$ is given by the coefficient of $\alpha_{\mathrm{s}} / 4 \pi$ of eq. A.3 in ref. [32].
} 


\subsection{NNLL' formulæ}

Finally, we examine the necessary ingredients for NNLL' accuracy. As discussed in section 2.5.2, we obtain all the logarithmic contributions of the soft functions at $\mathcal{O}\left(\alpha_{s}^{2}\right)$ by RG evolution. The two-loop hard functions and the $\delta\left(\mathcal{T}_{0}\right)$ contributions of the soft functions at $\mathcal{O}\left(\alpha_{s}^{2}\right)$ must instead be calculated explicitly.

Writing the additional $\mathcal{O}\left(\alpha_{\mathrm{s}}^{2}\right)$ term needed to reach full NNLL' accuracy as

$$
\frac{\mathrm{d} \sigma^{\mathrm{NNLL}}}{\mathrm{d} \Phi_{0} \mathrm{~d} \tau_{B}}=\frac{\mathrm{d} \sigma^{\mathrm{NNLL}}}{\mathrm{d} \Phi_{0} \mathrm{~d} \tau_{B}}+\frac{\mathrm{d} \sigma^{\mathrm{NNLL}} \alpha_{s}^{2}}{\mathrm{~d} \Phi_{0} \mathrm{~d} \tau_{B}}
$$

we find

$$
\begin{aligned}
& \frac{\mathrm{d} \sigma^{\mathrm{NNLL}_{\alpha_{s}^{2}}}}{\mathrm{~d} \Phi_{0} \mathrm{~d} \tau_{B}}=U^{\mathrm{NLL}}\left(\mu_{h}, \mu_{B}, \mu_{s}, L_{h}, L_{s}\right) \\
& \times\left\{\left[\frac{\alpha_{s}^{2}\left(\mu_{h}\right)}{16 \pi^{2}} \operatorname{Tr}\left\{\mathbf{u}^{\mathrm{NLL}}\left(\beta_{t}, \theta, \mu_{h}, \mu_{s}\right) \mathbf{H}^{(2)}\left(M, \beta_{t}, \theta, \mu_{h}\right) \mathbf{u}^{\dagger \mathrm{NLL}}\left(\beta_{t}, \theta, \mu_{h}, \mu_{s}\right) \mathbf{s}^{(0)}\right\}\right.\right. \\
& +\frac{\alpha_{s}^{2}\left(\mu_{s}\right)}{16 \pi^{2}} \operatorname{Tr}\left\{\mathbf{u}^{\mathrm{NLL}}\left(\beta_{t}, \theta, \mu_{h}, \mu_{s}\right) \mathbf{H}^{(0)}\left(M, \beta_{t}, \theta, \mu_{h}\right) \mathbf{u}^{\dagger \mathrm{NLL}}\left(\beta_{t}, \theta, \mu_{h}, \mu_{s}\right) \tilde{\mathbf{S}}_{B}^{(2)}\left(\partial_{\eta_{s}}+L_{s}, \beta_{t}, \theta, \mu_{s}\right)\right\} \\
& +\frac{\alpha_{s}\left(\mu_{h}\right) \alpha_{s}\left(\mu_{s}\right)}{16 \pi^{2}} \operatorname{Tr}\left\{\mathbf{u}^{\mathrm{NLL}}\left(\beta_{t}, \theta, \mu_{h}, \mu_{s}\right) \mathbf{H}^{(1)}\left(M, \beta_{t}, \theta, \mu_{h}\right) \mathbf{u}^{\dagger \mathrm{NLL}}\left(\beta_{t}, \theta, \mu_{h}, \mu_{s}\right)\right. \\
& \left.\left.\times \tilde{\mathbf{S}}_{B}^{(1)}\left(\partial_{\eta_{s}}+L_{s}, \beta_{t}, \theta, \mu_{s}\right)\right\}\right] \times \tilde{B}_{a}^{(0)}\left(z_{a}, \mu_{B}\right) \tilde{B}_{b}^{(0)}\left(z_{b}, \mu_{B}\right) \\
& +\frac{\alpha_{s}^{2}\left(\mu_{B}\right)}{16 \pi^{2}} \operatorname{Tr}\left\{\mathbf{u}^{\mathrm{NLL}}\left(\beta_{t}, \theta, \mu_{h}, \mu_{s}\right) \mathbf{H}^{(0)}\left(M, \beta_{t}, \theta, \mu_{h}\right) \mathbf{u}^{\dagger \mathrm{NLL}}\left(\beta_{t}, \theta, \mu_{h}, \mu_{s}\right) \mathbf{s}^{(0)}\right\} \\
& \times\left(\tilde{B}_{a}^{(2)}\left(\partial_{\eta_{B}}+L_{B}, z_{a}, \mu_{B}\right) \tilde{B}_{b}^{(0)}\left(z_{b}, \mu_{B}\right)+\tilde{B}_{a}^{(0)}\left(z_{a}, \mu_{B}\right) \tilde{B}_{b}^{(2)}\left(\partial_{\eta_{B}^{\prime}}+L_{B}, z_{b}, \mu_{B}\right)\right. \\
& \left.+\tilde{B}_{a}^{(1)}\left(\partial_{\eta_{B}}+L_{B}, z_{a}, \mu_{B}\right) \tilde{B}_{b}^{(1)}\left(\partial_{\eta_{B}^{\prime}}+L_{B}, z_{b}, \mu_{B}\right)\right) \\
& +\frac{\alpha_{s}\left(\mu_{h}\right) \alpha_{s}\left(\mu_{B}\right)}{16 \pi^{2}} \operatorname{Tr}\left\{\mathbf{u}^{\mathrm{NLL}}\left(\beta_{t}, \theta, \mu_{h}, \mu_{s}\right) \mathbf{H}^{(1)}\left(M, \beta_{t}, \theta, \mu_{h}\right) \mathbf{u}^{\dagger \mathrm{NLL}}\left(\beta_{t}, \theta, \mu_{h}, \mu_{s}\right) \mathbf{s}^{(0)}\right\} \\
& \times\left(\tilde{B}_{a}^{(1)}\left(\partial_{\eta_{B}}+L_{B}, z_{a}, \mu_{B}\right) \tilde{B}_{b}^{(0)}\left(z_{b}, \mu_{B}\right)+\tilde{B}_{a}^{(0)}\left(z_{a}, \mu_{B}\right) \tilde{B}_{b}^{(1)}\left(\partial_{\eta_{B}^{\prime}}+L_{B}, z_{b}, \mu_{B}\right)\right) \\
& +\frac{\alpha_{s}\left(\mu_{s}\right) \alpha_{s}\left(\mu_{B}\right)}{16 \pi^{2}} \operatorname{Tr}\left\{\mathbf{u}^{\mathrm{NLL}}\left(\beta_{t}, \theta, \mu_{h}, \mu_{s}\right) \mathbf{H}^{(0)}\left(M, \beta_{t}, \theta, \mu_{h}\right) \mathbf{u}^{\dagger \mathrm{NLL}}\left(\beta_{t}, \theta, \mu_{h}, \mu_{s}\right)\right. \\
& \left.\times \tilde{\mathbf{S}}_{B}^{(1)}\left(\partial_{\eta_{s}}+L_{s}, \beta_{t}, \theta, \mu_{s}\right)\right\} \\
& \left.\times\left(\tilde{B}_{a}^{(1)}\left(\partial_{\eta_{B}}+L_{B}, z_{a}, \mu_{B}\right) \tilde{B}_{b}^{(0)}\left(z_{b}, \mu_{B}\right)+\tilde{B}_{a}^{(0)}\left(z_{a}, \mu_{B}\right) \tilde{B}_{b}^{(1)}\left(\partial_{\eta_{B}^{\prime}}+L_{B}, z_{b}, \mu_{B}\right)\right)\right\} \\
& \times \frac{\eta_{\mathrm{tot}}^{\mathrm{NLL}}}{\tau_{B}^{1-\eta_{\mathrm{tot}}^{\mathrm{NLL}}}} \frac{e^{-\gamma_{E}} \eta_{\mathrm{tot}}^{\mathrm{NLL}}}{\Gamma\left(1+\eta_{\mathrm{tot}}^{\mathrm{NLL}}\right)} .
\end{aligned}
$$

In the previous equation, we only included the $\mathcal{O}\left(\alpha_{\mathrm{s}}^{2}\right)$ contributions coming from the boundary conditions of the hard, soft and beam functions evaluated at their characteristic 
scales. We neglect other contributions from e.g. the evolution matrices, which appear at the same order in the counting $\alpha_{\mathrm{s}} L \sim 1$, since these terms form part of the $\mathrm{N}^{3} \mathrm{LL}$ resummation.

At present, our implementation of eq. (3.16) misses the term in the second line since we have set the two-loop hard function coefficient $\mathbf{H}^{(2)}\left(M, \beta_{t}, \theta, \mu_{h}\right)=0$. The term in the third line is only missing the $\tilde{\mathbf{S}}_{B}^{(2,0)}$ (cfr. eq. (2.28)) contributions to the two-loop soft functions $\tilde{\mathbf{S}}_{B}^{(2)}\left(\partial_{\eta_{s}}+L_{s}, \beta_{t}, \theta, \mu_{s}\right)$; these are completely unknown at present and hence have also been set to zero. We have, however, included the known two-loop contributions to the beam functions. As a result, we cannot yet claim full NNLL' accuracy. We define our results obtained dropping the aforementioned two-loop hard and soft contributions proportional to $\delta\left(\mathcal{T}_{0}\right)$ as an approximate next-to-next-to-leading logarithmic accuracy (NNLL $\mathrm{N}_{\mathrm{a}}^{\prime}$ ) - this expression is nevertheless sufficient to match the resummed piece to the NLO fixed order calculations for $t \bar{t}+$ jet production. Indeed, when the evolution is switched off (i.e. set to unity), the formula above reproduces the complete singular spectrum for $\mathcal{T}_{0}>0$, valid in the limit $\mathcal{T}_{0} \ll M$.

\section{Numerical results}

We now present numerical results from the resummation framework developed in section 3 . We have implemented eq. (3.12), eq. (3.14) and eq. (3.16) in the GENEvA code, as well as the NLO fixed order calculations for $t \bar{t}$ and $t \bar{t}+$ jet. This allows us to study both the resummed distributions and the matching of the resummed calculation to the fixed order. For sake of definiteness, all the results presented in this section have been obtained for $p p$ collisions at a centre-of-mass energy of $\sqrt{S}=13 \mathrm{TeV}$ and using PDF4LHC15_nnlo parton distribution functions from LHAPDF $[75,76]$. The central predictions have been obtained running all scales to a common scale $\mu$ equal to the $t \bar{t}$ invariant mass $M$. In all figures present in this section, the statistical uncertainties associated with the Monte Carlo integrations are reported, when visible, as vertical error bars. We estimate the theoretical uncertainties for the fixed order predictions by varying the central choice for $\mu_{R}=\mu_{F}=M$ up and down by a factor of two and take the maximal absolute deviation from the central result as the fixed order uncertainty.

We begin by verifying that the approximate fixed order expressions, which we obtain from the resummed calculation by setting the various resummation scales equal to the hard scale, are able to reproduce the behaviour of the full fixed order calculation as $\mathcal{T}_{0} \rightarrow 0$. Comparisons of the full with the approximate fixed order results are shown in figure 1 at $\mathrm{LO}_{1}$ (i.e. $\mathrm{LO} t \bar{t}+$ jet) and $\mathrm{NLO}_{1}$ accuracy. We observe that, for small values of $\mathcal{T}_{0} \lesssim 10^{-1} \mathrm{GeV}$, the approximate FO reproduces the behaviour of the full calculation very well, both for the central values and the scale variations. This gives us confidence that the factorisation theorem is valid and that our calculation of the finite part of the one-loop soft function is correct. We notice that when the full $\mathrm{NLO}_{1}$ result crosses zero in the right plot, the associated statistical errors grow large, resulting in a instability in the ratio plot shown in the lower panel.

Before studying the resummed result, we have to provide a procedure to turn off the resummation before the exponentiated singular terms become too large, spoiling the 

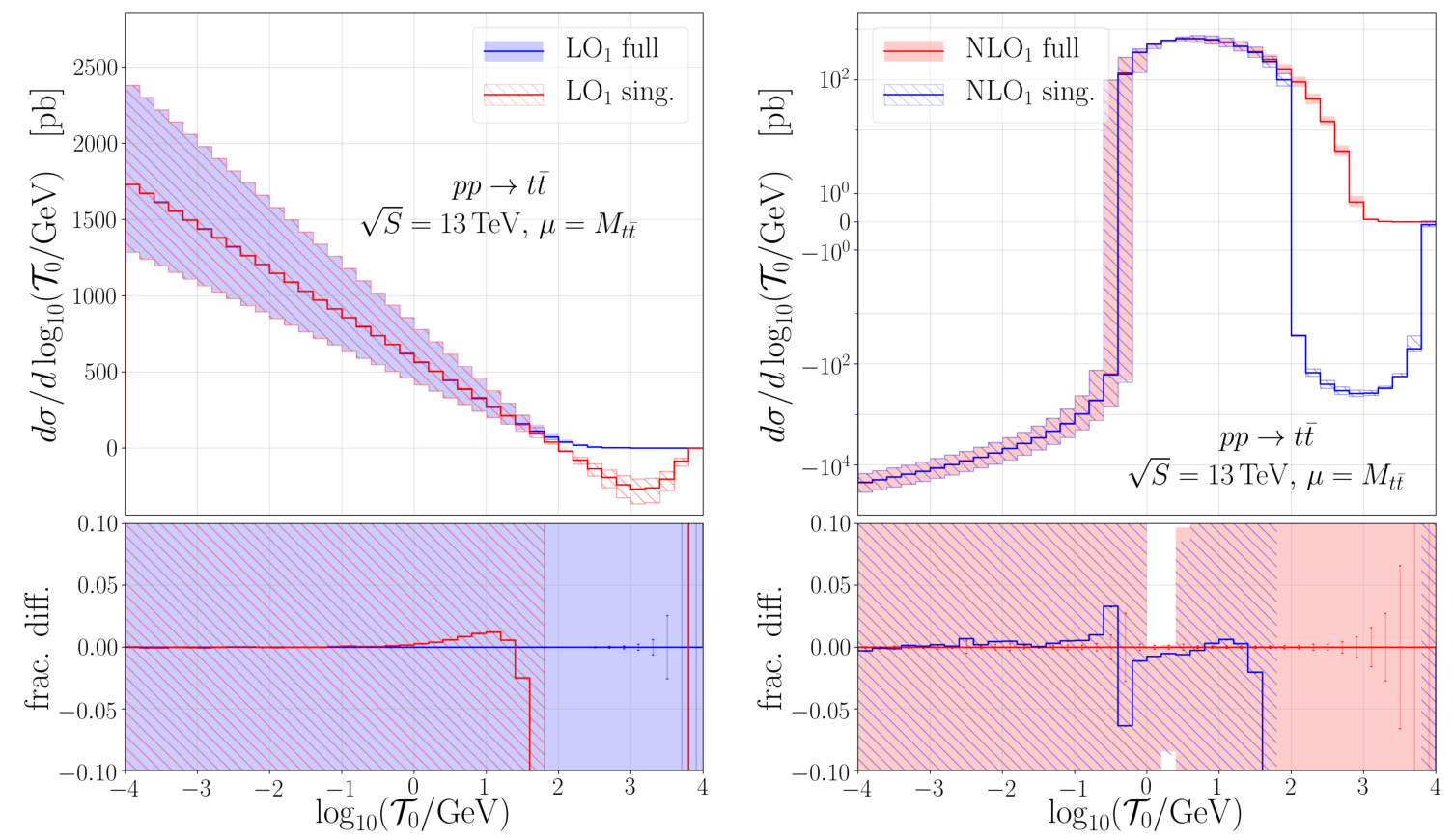

Figure 1. Approximate fixed order results for the $\mathcal{T}_{0}$ distribution obtained from our factorisation theorem compared with full calculations at LO (left) and NLO (right). The approximate results correctly reproduce the fixed order behaviour in the $\mathcal{T}_{0} \rightarrow 0$ limit.

predictions in the fixed order region. We do so in a smooth fashion by employing the profile scales introduced in refs. [64, 77, 78]. These profiles evolve the beam and soft scales to the hard scale as a function of $\tau_{B}$ and hence stop the RG evolution and resummation when the common scale $\mu_{\mathrm{NS}}=\mu_{S}=\mu_{B}=\mu_{H}$ is reached. Specifically, the profiles take the form:

$$
\begin{aligned}
\mu_{H} & =\mu_{\mathrm{NS}}, \\
\mu_{S}\left(\mathcal{T}_{0}\right) & =\mu_{\mathrm{NS}} f_{\text {run }}\left(\mathcal{T}_{0} / M\right), \\
\mu_{B}\left(\mathcal{T}_{0}\right) & =\mu_{\mathrm{NS}} \sqrt{f_{\text {run }}\left(\mathcal{T}_{0} / M\right)},
\end{aligned}
$$

where the common profile function $f_{\text {run }}(y)$ is given by [79]

$$
f_{\text {run }}(y)= \begin{cases}y_{0}\left[1+\left(y / y_{0}\right)^{2} / 4\right] & y \leq 2 y_{0} \\ y & 2 y_{0} \leq y \leq y_{1} \\ y+\frac{\left(2-y_{2}-y_{3}\right)\left(y-y_{1}\right)^{2}}{2\left(y_{2}-y_{1}\right)\left(y_{3}-y_{1}\right)} & y_{1} \leq y \leq y_{2} \\ 1-\frac{\left(2-y_{1}-y_{2}\right)\left(y-y_{3}\right)^{2}}{2\left(y_{3}-y_{1}\right)\left(y_{3}-y_{2}\right)} & y_{2} \leq y \leq y_{3} \\ 1 & y_{3} \leq y\end{cases}
$$

This functional form ensures the canonical scaling behaviour for values below $y_{1}$ and turns off the resummation above $y_{3}$. In order to determine the parameters $y_{i}$ of the profiles, it is instructive to examine the behaviour of the singular and nonsingular contributions to the cross section as a function of $\tau_{B}$ relative to the fixed order calculation. This is shown at $\mathrm{LO}_{1}$ 

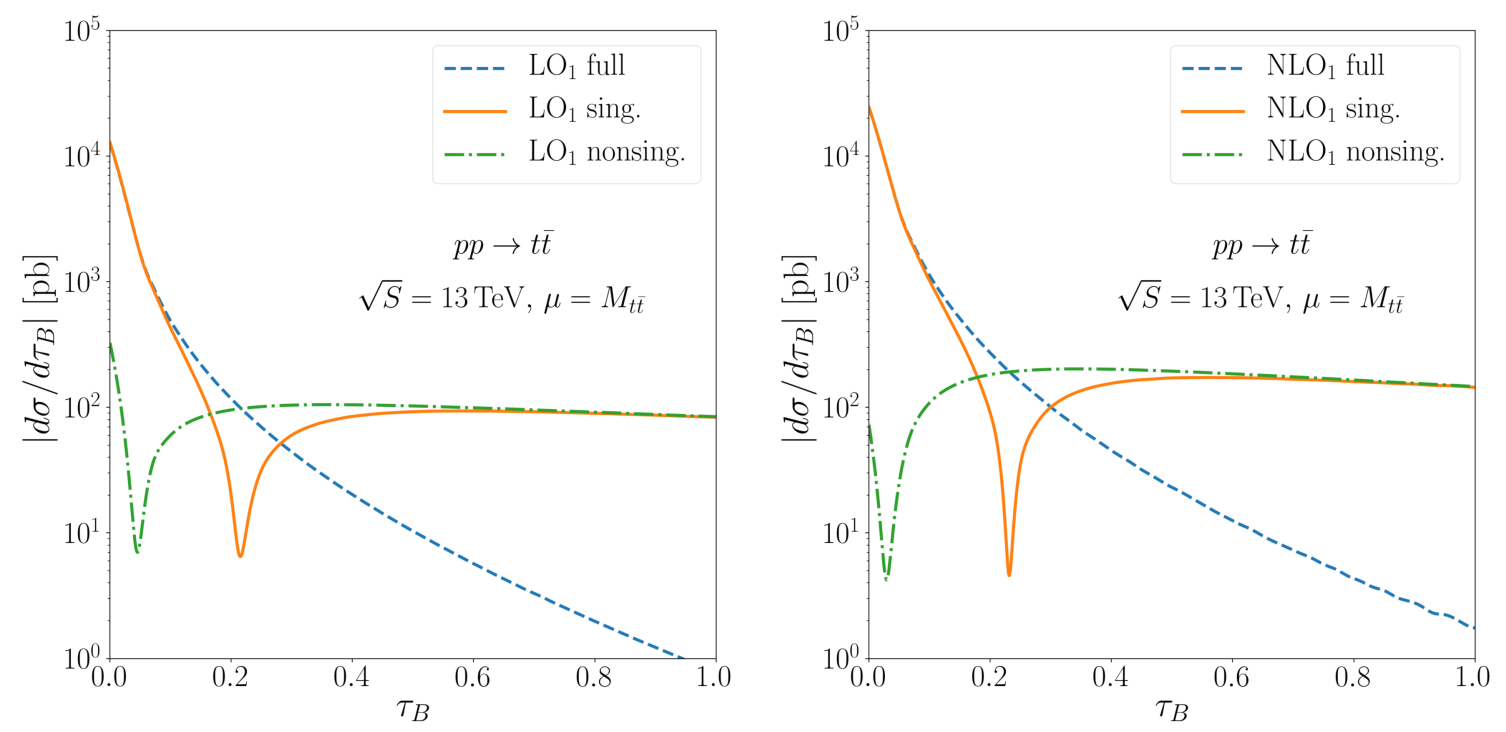

Figure 2. Comparison of the absolute values for the singular and nonsingular contributions to the $\mathcal{T}_{0}$ distribution with fixed order results at LO (left) and NLO (right) accuracy.

and $\mathrm{NLO}_{1}$ accuracy in figure 2. We see that the singular contribution to the cross section becomes of a similar size to the fixed order when $\tau_{B}$ is just above 0.2 . The behaviour at different orders is very similar. We therefore make the choices

$$
y_{0}=1.0 \mathrm{GeV} / M, \quad\left\{y_{1}, y_{2}, y_{3}\right\}=\{0.1,0.175,0.25\} .
$$

We now discuss the resummed results. In order to estimate the theoretical uncertainties, we vary the central choices for the profile scales in eq. (4.1) independently while keeping the hard scale fixed. This gives us four independent variations. In addition, we consider two more profile functions where we shift all the $y_{i}$ transition points together by \pm 0.015 while keeping all of the scales fixed at their central values. Hence, we obtain in total six profile variations. We consider the maximal absolute deviation in the results with respect to the central prediction as the resummation uncertainty.

In figure 3 , we show the peak region of the resummed $\mathcal{T}_{0}$ distribution. We compare predictions at different primed and unprimed levels of accuracy from NLL to NNLL ${ }_{a}^{\prime}$. Examining the unprimed results, we see a large shift in the central value between the NLL and NNLL results, though the central prediction for the NNLL result remains within the scale uncertainty band of lower order calculation. We also observe that the size of the band does not reduce substantially when moving from one order to the next. On the other hand, comparing the NLL' and NNLL ${ }_{a}^{\prime}$ results we observe both a more stable central value and also a sizeable reduction of the theoretical uncertainties. This highlights the need for full NNLL' accuracy in this process, which we hope to report on in future work.

As mentioned in section 3.2, for the production of coloured particles there is a certain amount of ambiguity in whether one should expand terms or instead keep them inside the exponential prefactor. This ambiguity starts at NNLL accuracy, since these terms are the first to contribute at $\mathcal{O}\left(\alpha_{\mathrm{s}}\right)$ in the logarithmic counting of the exponent. Indeed, while it is 

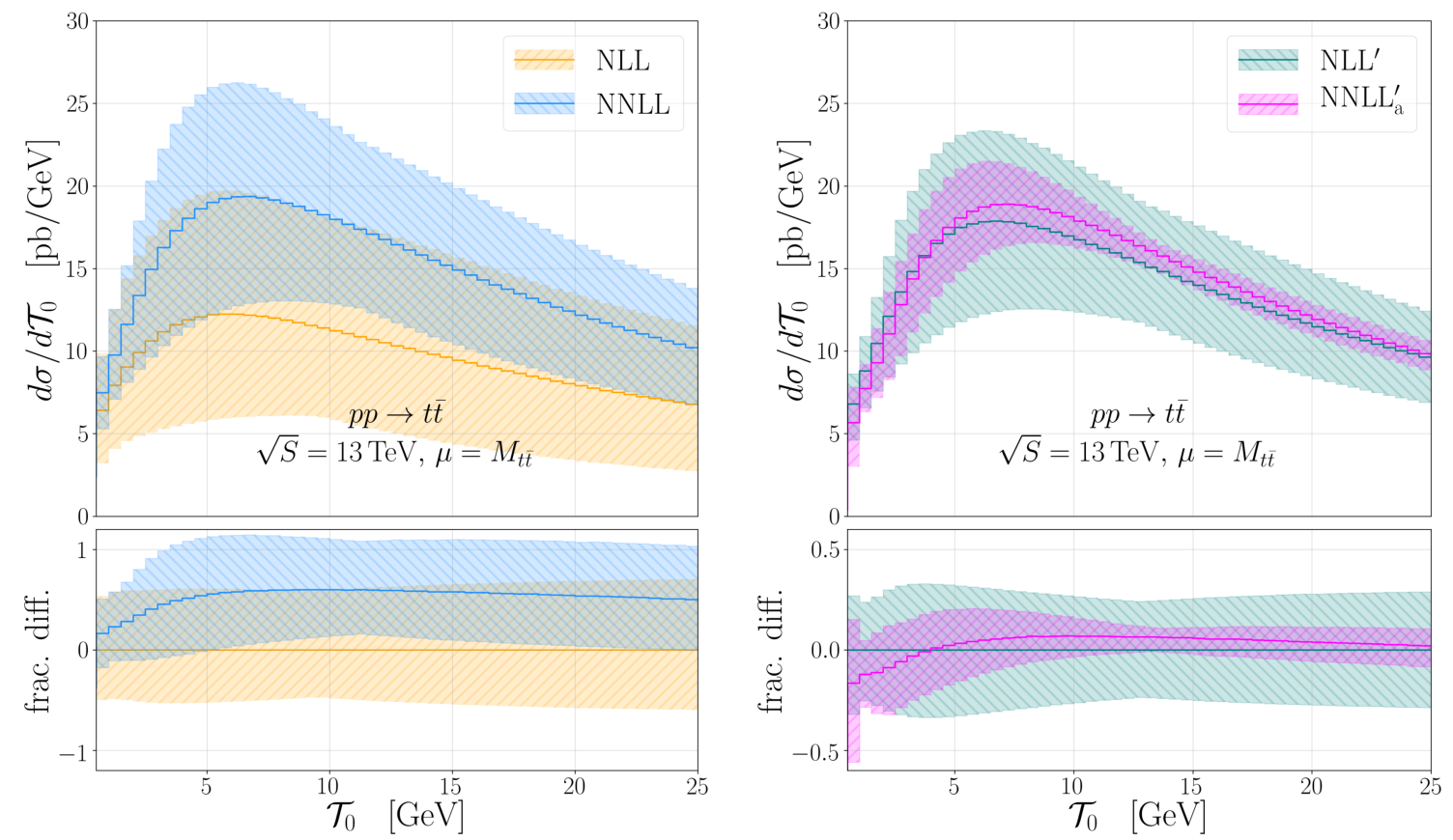

Figure 3. Resummed $\mathcal{T}_{0}$ distribution at successive unprimed (left) and primed (right) orders. Compared to the full NNLL' result, the approximate NNLL' prediction shown on the right misses only finite $\mathcal{O}\left(\alpha_{\mathrm{s}}^{2}\right)$ terms proportional to $\delta\left(\mathcal{T}_{0}\right)$ in the hard and soft functions.

necessary to evaluate the non-diagonal evolution matrix $\mathbf{u}$ as a perturbative expansion, the product between the diagonal evolution matrix $U$ and the generating function appearing e.g. in the first line of eq. (3.14) may be expanded in the same way or kept exact. We choose the former by default; however, it is interesting to assess the (formally higher order) effect of making the other choice. In figure 4, we compare the resummed distribution with and without this expansion, at both NNLL and NNLL a accuracy. We observe very little difference between the expanded and unexpanded results, suggesting that the effects of these missing higher order terms in the expanded results are minimal.

We now consider the matching of the resummed and fixed order calculations. We perform an additive matching, following the same spirit as recent GENEvA implementations (see e.g. ref. [49]). The appropriate combinations of resummed and fixed order accuracies are given in table 1 . The total perturbative uncertainty is calculated by adding in quadrature the previously discussed fixed order and resummation uncertainties. We define our matched spectrum as

$$
\frac{\mathrm{d} \sigma^{\text {match }}}{\mathrm{d} \mathcal{T}_{0}}=\frac{\mathrm{d} \sigma^{\text {resum }}}{\mathrm{d} \mathcal{T}_{0}}+\frac{\mathrm{d} \sigma^{\text {FO }}}{\mathrm{d} \mathcal{T}_{0}}-\left[\frac{\mathrm{d} \sigma^{\text {resum }}}{\mathrm{d} \mathcal{T}_{0}}\right]_{\text {FO }}
$$

where the final term removes double-counting between the resummed and fixed order pieces. In GENEVA implementations at NNLL'+NNLO, it acts as a subtraction term local in $\mathcal{T}_{0}$, which requires the fixed order calculation to use a $\mathcal{T}_{0}$-preserving mapping. This can have the positive feature of reducing the impact of fiducial power corrections compared to a simple slicing approach $[80,81]$. 

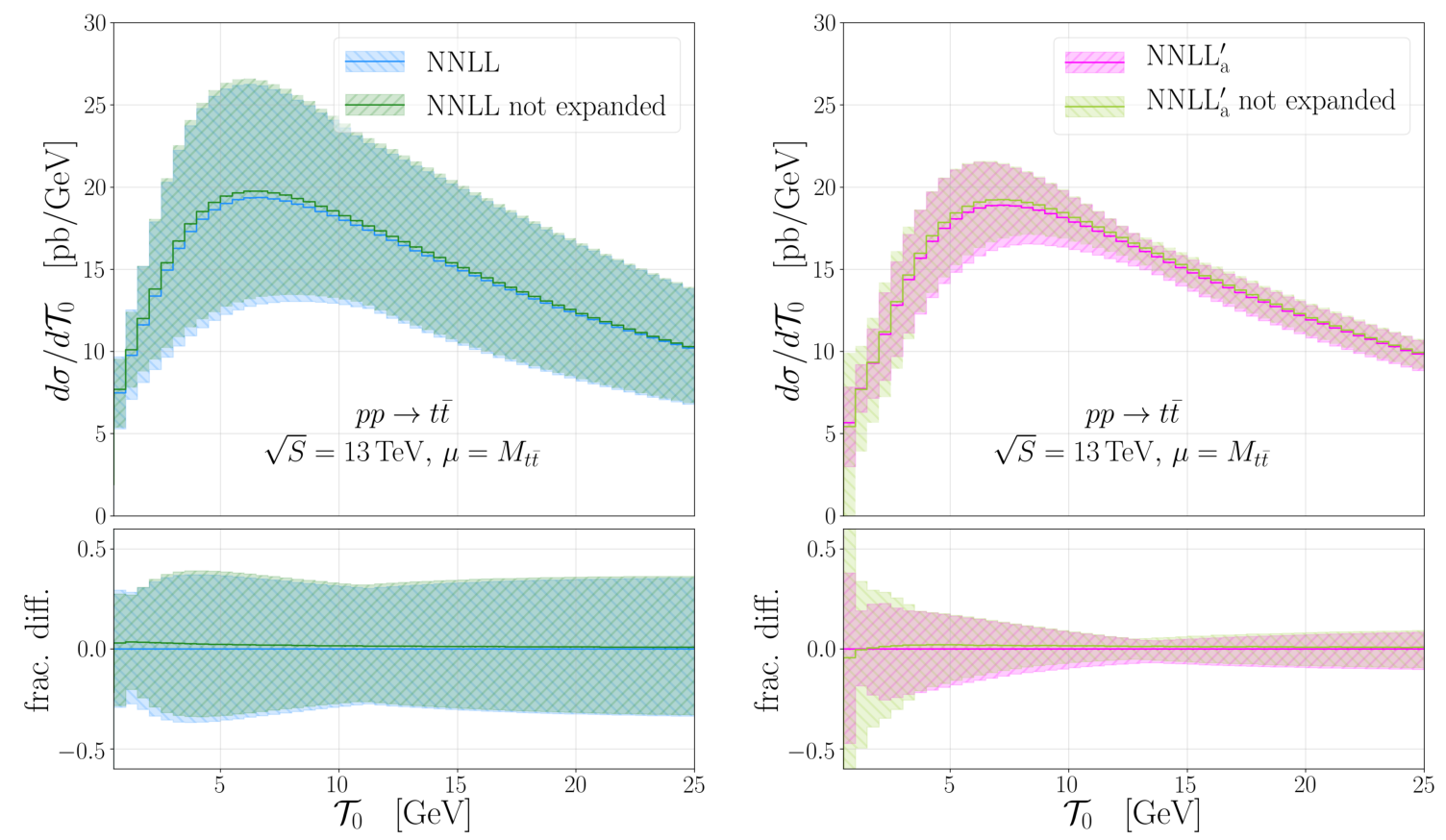

Figure 4. Resummed $\mathcal{T}_{0}$ distribution with and without the expansion of $U$ in eq. (3.2), at both NNLL (left) and NNLL' accuracy (right).

Finally, in figure 5 we present our best predictions across the whole spectrum. In order to highlight the effect of these higher-order corrections we show the resummed results at various resummation orders matched to the appropriate fixed order calculations. We divide the spectrum into the peak region, where resummation effects are most important, the transition, where resummed and fixed order contributions compete for importance, and the tail, where the fixed order is dominant. Examining the peak region, we notice slightly larger uncertainty bands for the NNLL $+\mathrm{LO}_{1}$ compared to the $\mathrm{NLL}^{\prime}+\mathrm{LO}_{1}$. The uncertainty bands are, however, significantly reduced once $\mathrm{NNLL}_{\mathrm{a}}^{\prime}+\mathrm{NLO}_{1}$ accuracy is reached. In the transition and tail regions, a clear difference between the $\mathrm{NNLL}_{\mathrm{a}}^{\prime}+\mathrm{NLO}_{1}$ and the lower order results emerges above $\sim 60 \mathrm{GeV}$ due to the additional contributions of the $\mathrm{NLO}_{1}$ calculation.

\section{Conclusions}

In this work, we studied the resummation of the zero-jettiness variable $\mathcal{T}_{0}$ in the context of top-quark pair production at hadron colliders. Starting from the frameworks of the Soft-Collinear and Heavy-Quark effective theories, we derived a factorisation formula for the process, which allowed us to express the resummed cross section at any logarithmic order in terms of hard, soft and beam functions. We then proceeded to calculate the relevant soft functions at one-loop order, which is a necessary boundary condition for reaching NLL' accuracy. Taking advantage of the known behaviour of the large logarithms for each of the ingredients of the factorisation theorem under renormalisation group evolution, we derived final expressions for the $\mathcal{T}_{0}$ distribution at $\mathrm{NLL}^{\prime}$, NNLL and approximate NNLL' accuracy, 

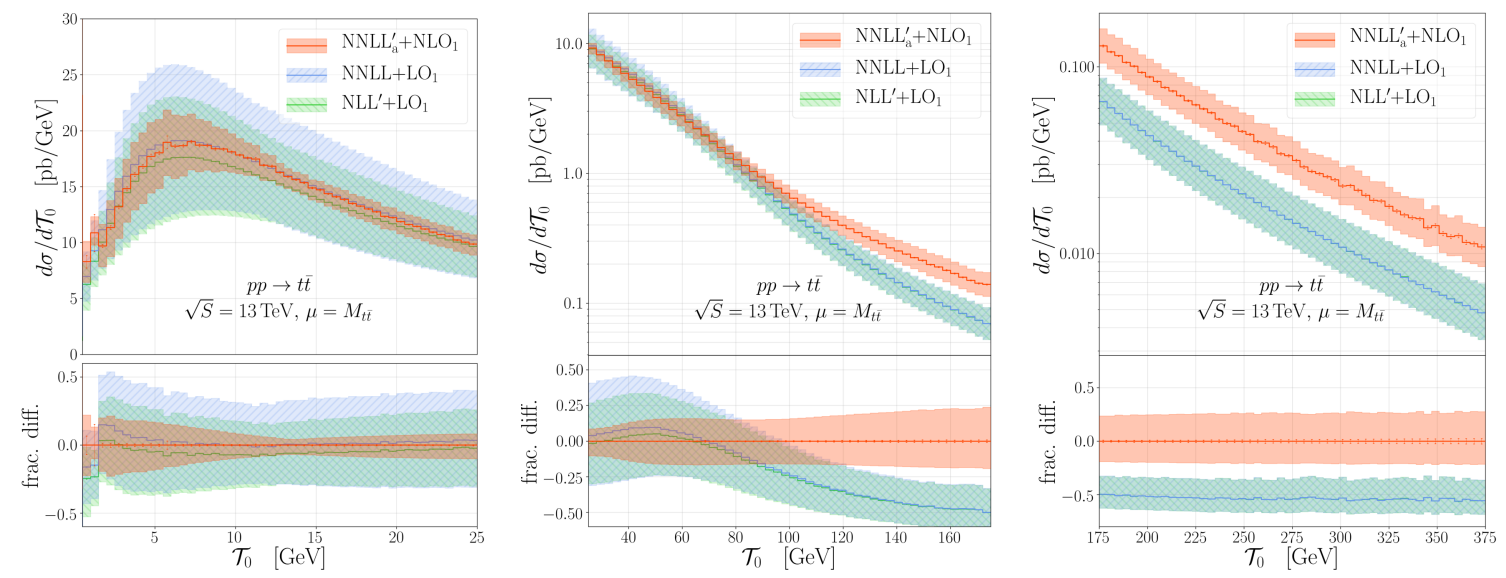

Figure 5. Resummed predictions matched to the appropiate fixed order for the $\mathcal{T}_{0}$ distribution at increasing accuracy in the peak (left), transition (centre) and tail (right) regions.

which we referred to as NNLL $L_{a}^{\prime}$. The approximation neglects the two-loop contributions to the hard and soft functions proportional to $\delta\left(\mathcal{T}_{0}\right)$, where the latter are presently unknown. We numerically evaluated our formulæ up to approximate NNLL' order, and matched our resummed predictions to fixed order calculations of the $t \bar{t}$ and $t \bar{t}+$ jet processes at NLO.

The work presented here achieves the highest accuracy matched calculation in a resolution variable for the $t \bar{t}$ process, and provides the foundation for a full NNLOPS implementation in the GenEva framework. In the future, resummed calculations for other resolution variables (such as the transverse momentum of the $t \bar{t}$ pair) at this accuracy could also be used in GENEva to examine the effect of the choice of resolution variable on NNLOPS predictions for this process, as was done for Drell-Yan in ref. [45].

At present, two ingredients are missing from our implementation which prevent us from reaching full NNLL' accuracy - the colour-decomposed hard function at NNLO, and the term of the two-loop soft function proportional to $\delta\left(\mathcal{T}_{0}\right)$. The first of these can, in principle, be constructed from ingredients already available in the literature — for example, the necessary two-loop amplitudes in colour-decomposed form were published in ref. [69] in the form of interpolation tables. The second, soft piece, could either be calculated analytically or be obtained using automated numerical tools such as SoftSERVE [82-84]. We intend to explore the effect of incorporating these additional terms on our results in the near future.

The factorisation theorem which we derived in this work is also applicable to other processes which involve the production of heavy coloured particles, such as the associated production of a heavy quark pair with Higgs or electroweak bosons. The main obstacle in reaching NNLL' accuracy for these processes is the availability of two-loop hard and soft functions. Nevertheless, in order to achieve the approximate NNLL $\mathrm{a}_{\mathrm{a}}^{\prime}$ accuracy used for our results, one can instead make use of the one-loop hard functions which are available in the literature [85-88]. The one loop soft functions must be calculated anew, allowing for a more general kinematic dependence. 


\section{Acknowledgments}

We are grateful to S. Kallweit, R. Nagar and D. Napoletano for useful discussions. We thank A. Gavardi, G. Marinelli and F. Tackmann for a careful reading of and comments on the manuscript. The work of SA, AB and MAL is supported by the ERC Starting Grant REINVENT-714788. SA acknowledges funding from Fondazione Cariplo and Regione Lombardia, grant 2017-2070 and from MIUR through the FARE grant R18ZRBEAFC. MAL is also supported by the Deutsche Forschungsgemeinschaft (DFG) under Germany's Excellence Strategy - EXC 2121 "Quantum Universe" — 390833306. We acknowledge the CINECA award under the ISCRA initiative and the National Energy Research Scientific Computing Center (NERSC), a U.S. Department of Energy Office of Science User Facility operated under Contract No. DEAC02-05CH11231, for the availability of the high performance computing resources needed for this work.

\section{A Derivation of the factorisation formula}

In this appendix we discuss the details of the derivation of the factorisation formula for the top-quark pair production process in the limit of small $\mathcal{T}_{0}$ by employing position space SCET and the multipole expansion.

We begin with some definitions which will be needed throughout this appendix. We work in the centre-of-mass frame of the hadronic collisions and introduce two light-like vectors $n_{a}$ and $n_{b}$ along the beam directions so that the momenta of the incoming protons are given by $P_{a, b}^{\mu}=E_{\mathrm{cm}} n_{a, b}^{\mu} / 2$. These vectors satisfy the relations $n_{b}=\bar{n}_{a}=\left(1,-\vec{n}_{a}\right)$ and $n_{a} \cdot n_{b}=2$.

Neglecting all complications related to Glauber modes, the description of the top-quark pair production process within an effective field theory framework requires both SCET, to describe the interactions of the collinear fields with the soft gluons, and Heavy-Quark Effective Theory (HQET), to describe the soft interactions of the heavy quark fields. When the heavy-quark fields $h_{v}(x)$ appear in combination with collinear (anti-collinear) fields, they must be Taylor expanded around $x^{-}\left(x^{+}\right)$since the residual $x$ variations of these fields are identical to those of ultrasoft fields. The effective theory operators which are relevant for this process contain two heavy quark fields, one collinear and one anti-collinear field: the double projection onto plus and minus components therefore implies that the heavy quark fields must be evaluated at the origin. While the final state heavy quarks by definition do not contribute to the radiation in any hemisphere, the soft Wilson lines along the directions $v_{3}$ and $v_{4}$, which arise from the decoupling of the soft gluon interactions from the heavy quark fields, contribute instead to the hemisphere radiation measurements. The effective Hamiltonian for this process reads [32]

$$
\mathcal{H}_{\mathrm{eff}}(x)=\sum_{I, m} \int \mathrm{d} r \mathrm{~d} t e^{i m_{t}\left(v_{3}+v_{4}\right) \cdot x}\left[C_{I m}^{g g}(r, t) O_{I m}^{g g}(x, r, t)+C_{I m}^{q \bar{q}}(r, t) O_{I m}^{q \bar{q}}(x, r, t)+(q \leftrightarrow \bar{q})\right],
$$


where $I$ labels the colour and $m$ the Dirac structures. The operators explicitly read

$$
\begin{aligned}
& O_{I m}^{q \bar{q}}(x, r, t)=\sum_{\{a\}}\left(c_{I}^{q \bar{q}}\right)_{\{a\}} \bar{\chi}_{\bar{c}}^{a_{2}}\left(x+r n_{a}\right) \Gamma_{m}^{\prime} \chi_{c}^{a_{1}}\left(x+t n_{b}\right) \bar{h}_{v_{3}}^{a_{3}}(x) \Gamma_{m}^{\prime \prime} h_{v_{4}}^{a_{4}}(x) \\
& O_{I m}^{g g}(x, r, t)=\sum_{\{a\}}\left(c_{I}^{g g}\right)_{\{a\}} \mathcal{A}_{\bar{c} \nu \perp}^{a_{2}}\left(x+r n_{a}\right) \mathcal{A}_{c \mu \perp}^{a_{1}}\left(x+t n_{b}\right) \bar{h}_{v_{3}}^{a_{3}}(x) \Gamma_{m}^{\mu \nu} h_{v_{4}}^{a_{4}}(x),
\end{aligned}
$$

where the fields $\chi_{c}$ and $\mathcal{A}_{c}$ are the collinear gauge-invariant, collinear building blocks

$$
\chi_{c}=W_{c}^{\dagger}(x) \xi_{c}(x), \quad \mathcal{A}_{c \perp}^{\mu}=W_{c}^{\dagger}(x)\left(i D_{\perp}^{\mu} W_{c}(x)\right),
$$

with $\xi_{c}=\left(\hbar_{a} \hbar_{a} / 4\right) \psi(x)$. Similar equations hold for the anti-collinear quark $\chi_{\bar{c}}$ and gluon $\mathcal{A}_{\bar{c}}^{\mu}$ building blocks. The structures $\Gamma_{m}^{\mu \nu}, \Gamma_{m}^{\prime}$ and $\Gamma_{m}^{\prime \prime}$ span all relevant combinations of Dirac matrices and external vectors. The $c_{I}$ tensors define the orthogonal colour basis

$$
\begin{array}{ll}
\left(c_{1}^{q \bar{q}}\right)_{\{a\}}=\delta_{a_{1} a_{2}} \delta_{a_{3} a_{4}}, & \left(c_{2}^{q \bar{q}}\right)_{\{a\}}=t_{a_{2} a_{1}}^{c} t_{a_{3} a_{4}}^{c}, \\
\left(c_{1}^{g g}\right)_{\{a\}}=\delta^{a_{1} a_{2}} \delta_{a_{3} a_{4}}, & \left(c_{2}^{g g}\right)_{\{a\}}=i f^{a_{1} a_{2} c} t_{a_{3} a_{4}}^{c}, \quad\left(c_{3}^{g g}\right)_{\{a\}}=d^{a_{1} a_{2} c} t_{a_{3} a_{4}}^{c}
\end{array}
$$

where $\{a\}=\left\{a_{1}, a_{2}, a_{3}, a_{4}\right\}$ is a set of colour indices which can be either in the fundamental or adjoint representation depending on the field with which they are contracted. It is convenient to decouple the soft gluon interactions via the BPS (decoupling) transformations [89] for the collinear and heavy quark fields and obtain for the $q \bar{q}$ channel

$$
O_{I m}^{q \bar{q}}(x, r, t)=\sum_{\{a\},\{b\}}\left(c_{I}^{q \bar{q}}\right)_{\{a\}}\left[O_{m}^{h}(x)\right]^{b_{3} b_{4}}\left[O_{m}^{c}(x, r, t)\right]^{b_{1} b_{2}}\left[O^{s}(x)\right]^{\{a\},\{b\}},
$$

where

$$
\begin{aligned}
{\left[O_{m}^{h}(x)\right]^{b_{3} b_{4}} } & =\bar{h}_{v_{3}}^{b_{3}}(x) \Gamma_{m}^{\prime \prime} h_{v_{4}}^{b_{4}}(x), \quad\left[O_{m}^{c}(x, r, t)\right]^{b_{1} b_{2}}=\bar{\chi}_{\bar{c}}^{b_{2}}\left(x+r n_{a}\right) \Gamma_{m}^{\prime} \chi_{c}^{b_{1}}\left(x+t n_{b}\right), \\
{\left[O^{s}(x)\right]^{\{a\},\{b\}} } & =\left[Y_{v_{3}}^{\dagger}(x)\right]^{b_{3} a_{3}}\left[Y_{v_{4}}(x)\right]^{a_{4} b_{4}}\left[Y_{n_{b}}^{\dagger}(x)\right]^{b_{2} a_{2}}\left[Y_{n_{a}}(x)\right]^{a_{1} b_{1}}
\end{aligned}
$$

and the soft Wilson lines are defined as

$$
\begin{aligned}
& {\left[Y_{\left.n_{\{a, b\}}(x)\right]^{c d}}=\mathcal{P} \exp \left(i g_{s} \int_{-\infty}^{0} \mathrm{~d} t n_{\{a, b\}} \cdot A_{s}^{e}\left(x+t n_{\{a, b\}}\right) t_{c d}^{e}\right),\right.} \\
& {\left[Y_{\left.v_{\{3,4\}}(x)\right]^{c d}}=\mathcal{P} \exp \left(-i g_{s} \int_{0}^{\infty} \mathrm{d} t v_{\{3,4\}} \cdot A_{s}^{e}\left(x+t v_{\{3,4\}}\right) t_{c d}^{e}\right) .\right.}
\end{aligned}
$$

For the gluon fusion channel we have instead

$$
O_{I m}^{g g}(x, r, t)=\sum_{\{a\},\{b\}}\left(c_{I}^{g g}\right)_{\{a\}}\left[O_{m}^{h}(x)\right]_{b_{3} b_{4}}^{\mu \nu}\left[O^{c}(x, r, t)\right]_{\mu \nu}^{b_{1} b_{2}}\left[O^{s}(x)\right]^{\{a\},\{b\}},
$$

where

$$
\begin{aligned}
{\left[O_{m}^{h}(x)\right]_{b_{3} b_{4}}^{\mu \nu} } & =\bar{h}_{v_{3}}^{b_{3}}(x) \Gamma_{m}^{\mu \nu} h_{v_{4}}^{b_{4}}(x), \quad\left[O^{c}(x, r, t)\right]_{\mu \nu}^{b_{1} b_{2}}=\mathcal{A}_{c \mu \perp}^{b_{1}}\left(x+t n_{b}\right) \mathcal{A}_{\bar{c} \nu \perp}^{b_{2}}\left(x+r n_{a}\right) \\
{\left[O^{s}(x)\right]^{\{a\},\{b\}} } & =\left[Y_{v_{3}}^{\dagger}(x)\right]^{b_{3} a_{3}}\left[Y_{v_{4}}(x)\right]^{a_{4} b_{4}}\left[Y_{n_{b}}^{\text {adj } \dagger}(x)\right]^{b_{2} a_{2}}\left[Y_{n_{a}}^{\text {adj }}(x)\right]^{a_{1} b_{1}}
\end{aligned}
$$


and we have used the "adj" superscript for the Wilson line in the adjoint representation

$$
\left[Y_{n_{\{a, b\}}}^{\mathrm{adj}}(x)\right]^{c d}=\mathcal{P} \exp \left(i g_{s} \int_{-\infty}^{0} \mathrm{~d} t n_{\{a, b\}} \cdot A_{s}^{e}\left(x+t n_{\{a, b\}}\right)\left(-i f^{e c d}\right)\right) .
$$

We consider $B_{a}^{\mu}$ and $B_{b}^{\mu}$ to be the total final-state hadronic momenta in the hemispheres $a$ and $b$ and introduce their two components $B_{a}^{+}=n_{a} \cdot B_{a}, B_{b}^{+}=n_{b} \cdot B_{b}$. We evaluate the cross section differential in $B_{a}^{+}$and $B_{b}^{+}$in the limit where the radiation is soft or collinear

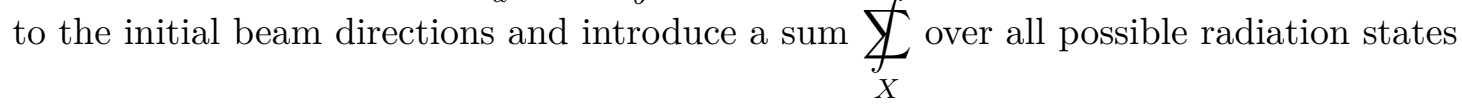

$$
\begin{aligned}
& \frac{\mathrm{d} \sigma}{\mathrm{d} B_{a}^{+} \mathrm{d} B_{b}^{+}}=\frac{1}{2 S} \int \frac{\mathrm{d}^{3} \vec{p}_{3}}{(2 \pi)^{3} 2 E_{3}} \int \frac{\mathrm{d}^{3} \vec{p}_{4}}{(2 \pi)^{3} 2 E_{4}} \underbrace{}_{X}(2 \pi)^{4} \delta^{(4)}\left(P_{a}+P_{b}-p_{3}-p_{4}-p_{X}\right) \\
& \times \frac{1}{4 d_{R}^{2}}\left|\left\langle t\left(p_{3}\right) \bar{t}\left(p_{4}\right) X\left(p_{X}\right)\left|\mathcal{H}_{\mathrm{eff}}(0)\right| P_{1}\left(P_{a}\right) P_{2}\left(P_{b}\right)\right\rangle\right|^{2} \\
& \times \delta\left(B_{a}^{+}-n_{a} \cdot B_{a}(X)\right) \delta\left(B_{b}^{+}-n_{b} \cdot B_{b}(X)\right),
\end{aligned}
$$

where the factor $1 / 4$ is due to the average over the initial state polarisations and the factor $d_{R}=\left\{N_{c}, N_{c}^{2}-1\right\}$ for the average over the colours of the initial state quarks or gluons. The hemisphere hadronic momenta $B_{a, b}^{\mu}(X)$ are obtained when the hemisphere momentum operators $\hat{p}_{a, b}^{\mu}$ act on the radiation states as

$$
\hat{p}_{a, b}^{\mu}|X\rangle=B_{a, b}^{\mu}(X)|X\rangle .
$$

We proceed by inserting in eq. (A.12) the identity

$$
1=\int \mathrm{d} M^{2} \mathrm{~d}^{4} q \delta^{(4)}\left(q-p_{3}-p_{4}\right) \delta\left(M^{2}-q^{2}\right),
$$

where $q^{\mu} \equiv p_{3}^{\mu}+p_{4}^{\mu}$ is the four momentum of the $t \bar{t}$ pair and we denote the invariant mass and the rapidity of the $t \bar{t}$ system by $M$ and $y_{t \bar{t}}$ respectively.

After a few manipulations we arrive at

$$
\begin{aligned}
& \frac{\mathrm{d} \sigma}{\mathrm{d} B_{a}^{+} \mathrm{d} B_{b}^{+}}=\frac{1}{16 S} \frac{1}{(2 \pi)^{2}} \int \mathrm{d} M \mathrm{~d} y_{t \bar{t}} \mathrm{~d} \cos \theta \mathrm{d} \phi_{t} M \beta_{t} \int \frac{\mathrm{d}^{2} \vec{q}_{\perp}}{(2 \pi)^{4}} \sum_{X}(2 \pi)^{4} \delta^{(4)}\left(P_{a}+P_{b}-q-p_{X}\right) \\
& \times \frac{1}{4 d_{R}^{2}}\left|\left\langle t\left(p_{3}\right) \bar{t}\left(p_{4}\right) X\left(p_{X}\right)\left|\mathcal{H}_{\text {eff }}(0)\right| P_{1}\left(P_{a}\right) P_{2}\left(P_{b}\right)\right\rangle\right|^{2} \delta\left(B_{a}^{+}-n_{a} \cdot B_{a}(X)\right) \delta\left(B_{b}^{+}-n_{b} \cdot B_{b}(X)\right),
\end{aligned}
$$

where $\beta_{t}$ and $\theta$ are defined in eq. (2.4) and $\phi_{t}$ is the azimuthal angle of the top quark $\left(v_{3}\right)$ in the $t \bar{t}$ rest frame. By employing the definition of the Dirac delta function,

$$
\delta^{(4)}\left(P_{a}+P_{b}-p_{3}-p_{4}-p_{X}\right)=\int \frac{\mathrm{d}^{4} x}{(2 \pi)^{4}} e^{i\left(P_{a}+P_{b}-p_{3}-p_{4}-p_{X}\right) \cdot x},
$$

the translation operator acting on the effective Hamiltonian

$$
\mathcal{H}_{\text {eff }}(x)=e^{+i \hat{P} \cdot \hat{x}} \mathcal{H}_{\mathrm{eff}}(0) e^{-i \hat{P} \cdot \hat{x}},
$$


and the momentum conservation relation, we rewrite the differential cross section as

$$
\begin{aligned}
& \frac{\mathrm{d} \sigma}{\mathrm{d} M \mathrm{~d} y_{t \bar{t}} \mathrm{~d} \cos \theta \mathrm{d} B_{a}^{+} \mathrm{d} B_{b}^{+}}= \\
& \frac{M \beta_{t}}{16 S} \frac{1}{(2 \pi)^{2}} \frac{1}{4 d_{R}^{2}} \int \mathrm{d} \phi_{t} \int \frac{\mathrm{d}^{2} \vec{q}_{\perp}}{(2 \pi)^{4}} \int \mathrm{d}^{4} x e^{+i\left(P_{a}+P_{b}-p_{3}-p_{4}-p_{X}\right) \cdot x} \\
& \times \sum_{X}\left\langle P_{1}\left(P_{a}\right) P_{2}\left(P_{b}\right)\left|e^{-i \hat{P} \cdot \hat{x}} \mathcal{H}_{\text {eff }}^{\dagger}(x) e^{i \hat{P} \cdot \hat{x}}\right| t\left(p_{3}\right) \bar{t}\left(p_{4}\right) X\left(p_{X}\right)\right\rangle \\
& \quad \times\left\langle t\left(p_{3}\right) \bar{t}\left(p_{4}\right) X\left(p_{X}\right)\left|\mathcal{H}_{\mathrm{eff}}(0)\right| P_{1}\left(P_{a}\right) P_{2}\left(P_{b}\right)\right\rangle \delta\left(B_{a}^{+}-n_{a} \cdot B_{a}(X)\right) \delta\left(B_{b}^{+}-n_{b} \cdot B_{b}(X)\right) \\
& =\frac{M \beta_{t}}{16 S} \frac{1}{(2 \pi)^{2}} \frac{1}{4 d_{R}^{2}} \int \mathrm{d} \phi_{t} \int \frac{\mathrm{d}^{2} \vec{q}_{\perp}}{(2 \pi)^{4}} \\
& \quad \times \int \mathrm{d}^{4} x\left\langle P_{1}\left(P_{a}\right) P_{2}\left(P_{b}\right)\left|\mathcal{H}_{\text {eff }}^{\dagger}(x)\right| t\left(p_{3}\right) \bar{t}\left(p_{4}\right)\right\rangle \delta\left(B_{a}^{+}-n_{a} \cdot \hat{p}_{a}\right) \\
& \quad \times \delta\left(B_{b}^{+}-n_{b} \cdot \hat{p}_{b}\right)\left\langle t\left(p_{3}\right) \bar{t}\left(p_{4}\right)\left|\mathcal{H}_{\mathrm{eff}}(0)\right| P_{1}\left(P_{a}\right) P_{2}\left(P_{b}\right)\right\rangle,
\end{aligned}
$$

where in the last line we have also expressed the delta function constraints in terms of the hemisphere momentum operators $\hat{p}_{a, b}^{\mu}$ which act on the radiation states as in eq. (A.13).

For the purpose of the derivation of the factorisation formula, we explicitly work out all the details for the gluon fusion channel contribution. The result for the quark anti-quark channel case can be obtained in a very similar way. After substituting the explicit expression for the second operator in eq. (A.2) one obtains

$$
\begin{aligned}
& \frac{\mathrm{d} \sigma^{g g}}{\mathrm{~d} M \mathrm{~d} y_{t \bar{t}} \mathrm{~d} \cos \theta \mathrm{d} B_{a}^{+} \mathrm{d} B_{b}^{+}}= \\
& \frac{M \beta_{t}}{32 S} \frac{1}{(2 \pi)^{4}} \frac{1}{4 d_{R}^{2}} \sum_{I, I^{\prime}, m, m^{\prime}} \int \mathrm{d} \phi_{t} \int \mathrm{d} x^{+} \mathrm{d} x^{-} e^{-i\left(q^{-} x^{+}+q^{+} x^{-}\right) / 2} \\
& \quad \times \int \mathrm{d}^{2} \vec{x}_{\perp} \delta^{(2)}\left(\vec{x}_{\perp}\right) \int \mathrm{d} r^{\prime} \mathrm{d} t^{\prime} \mathrm{d} r \mathrm{~d} t C_{I^{\prime} m^{\prime}}^{g g *}\left(r^{\prime}, t^{\prime}\right) C_{I m}^{g g}(r, t) \\
& \quad \times\left\langle P_{1}\left(P_{a}\right) P_{2}\left(P_{b}\right)\left|\sum_{\left\{a^{\prime}\right\},\left\{b^{\prime}\right\}}\left(c_{I^{\prime}}^{g g *}\right)_{\left\{a^{\prime}\right\}}\left[O_{m^{\prime}}^{h \dagger}(x)\right]_{b_{3}^{\prime} b_{4}^{\prime}}^{\rho \sigma}\left[O^{c \dagger}\left(x, r^{\prime}, t^{\prime}\right)\right]_{\rho \sigma}^{b_{1}^{\prime} b_{2}^{\prime}}\left[O^{s \dagger}(x)\right]^{\left\{a^{\prime}\right\},\left\{b^{\prime}\right\}}\right| t\left(p_{3}\right) \bar{t}\left(p_{4}\right)\right\rangle \\
& \quad \times \delta\left(B_{a}^{+}-n_{a} \cdot \hat{p}_{a}\right) \delta\left(B_{b}^{+}-n_{b} \cdot \hat{p}_{b}\right) \\
& \quad \times\left\langle t\left(p_{3}\right) \bar{t}\left(p_{4}\right)\left|\sum_{\{a\},\{b\}}\left(c_{I}^{g g}\right)_{\{a\}}\left[O_{m}^{h}(0)\right]_{b_{3} b_{4}}^{\mu \nu}\left[O^{c}(0, r, t)\right]_{\mu \nu}^{b_{1} b_{2}}\left[O^{s}(0)\right]^{\{a\},\{b\}}\right| P_{1}\left(P_{a}\right) P_{2}\left(P_{b}\right)\right\rangle .
\end{aligned}
$$

We define a vector of Wilson coefficients

$$
\left|C_{m}(r, t)\right\rangle=\sum_{I} C_{I m}(r, t)\left|c_{I}\right\rangle
$$

where the colour basis tensors in eq. (A.4) are understood as basis vectors $\left|c_{I}\right\rangle$ of an abstract colour space. We employ the colour space formalism to simplify the notation and rewrite 
the formula above as

$$
\begin{aligned}
& \frac{\mathrm{d} \sigma^{g g}}{\mathrm{~d} M \mathrm{~d} y_{t \bar{t}} \mathrm{~d} \cos \theta \mathrm{d} B_{a}^{+} \mathrm{d} B_{b}^{+}}= \\
& \frac{M \beta_{t}}{32 S} \frac{1}{(2 \pi)^{4}} \frac{1}{4 d_{R}^{2}} \sum_{m, m^{\prime}} \int \mathrm{d} \phi_{t} \int \mathrm{d} x^{+} \mathrm{d} x^{-} e^{-i\left(q^{-} x^{+}+q^{+} x^{-}\right) / 2} \\
& \quad \times \int \mathrm{d}^{2} \vec{x}_{\perp} \delta^{(2)}\left(\vec{x}_{\perp}\right) \int \mathrm{d} r^{\prime} \mathrm{d} t^{\prime} \mathrm{d} r \mathrm{~d} t \\
& \quad \times\left\langle C_{m^{\prime}}\left(r^{\prime}, t^{\prime}\right)\right|\left\langle P_{1}\left(P_{a}\right) P_{2}\left(P_{b}\right)\left|\left[O_{m^{\prime}}^{h \dagger}(x)\right]^{\rho \sigma}\left[O^{c \dagger}\left(x, r^{\prime}, t^{\prime}\right)\right]_{\rho \sigma}\left[\boldsymbol{O}^{s \dagger}(x)\right]\right| t\left(p_{3}\right) \bar{t}\left(p_{4}\right)\right\rangle \\
& \quad \times \delta\left(B_{a}^{+}-n_{a} \cdot \hat{p}_{a}\right) \delta\left(B_{b}^{+}-n_{b} \cdot \hat{p}_{b}\right) \\
& \quad \times\left\langle t\left(p_{3}\right) \bar{t}\left(p_{4}\right)\left|\left[O_{m}^{h}(0)\right]^{\mu \nu}\left[O^{c}(0, r, t)\right]_{\mu \nu}\left[\boldsymbol{O}^{s}(0)\right]\right| P_{1}\left(P_{a}\right) P_{2}\left(P_{b}\right)\right\rangle\left|C_{m}(r, t)\right\rangle .
\end{aligned}
$$

where the soft operator $\boldsymbol{O}^{s}(x)$ contains soft Wilson lines $\boldsymbol{Y}_{i}$ with the colour generators $\boldsymbol{T}_{i}$ acting in abstract colour space.

After the BPS (decoupling) transformation [89] the effective Lagrangian is written in terms of independent collinear, anti-collinear, heavy quark and soft Lagrangians which do not interact with each other. For this reason and the fact that the final state heavy quarks do not contribute to the radiation in any hemisphere, we can write the hemisphere momentum operators $\hat{p}_{a}$ and $\hat{p}_{b}$ as the sum of independent operators which act in the separate collinear, anti-collinear and soft sectors as [48]

$$
\hat{p}_{a}=\hat{p}_{a, n_{a}}+\hat{p}_{a, n_{b}}+\hat{p}_{a, s}, \quad \hat{p}_{b}=\hat{p}_{b, n_{a}}+\hat{p}_{b, n_{b}}+\hat{p}_{b, s} .
$$

The $n_{a}$ collinear sector cannot contribute to the momentum in the $n_{b}$ hemisphere and vice versa. Therefore $\hat{p}_{a, n_{b}}=\hat{p}_{b, n_{a}}=0$ and the relations above simplify to

$$
\hat{p}_{a}=\hat{p}_{n_{a}}+\hat{p}_{a, s}, \quad \hat{p}_{b}=\hat{p}_{n_{b}}+\hat{p}_{b, s},
$$

where $\hat{p}_{a, n_{a}}=\hat{p}_{n_{a}}$ and $\hat{p}_{b, n_{b}}=\hat{p}_{n_{b}}$ represent the total momentum operators of the collinear and anti-collinear sectors. We separate the soft and collinear contributions to the single hemispheres using the relations

$$
\begin{aligned}
\delta\left(B_{a}^{+}-n_{a} \cdot \hat{p}_{a}\right) & =\int \mathrm{d} b_{a}^{+} \mathrm{d} k_{a}^{+} \delta\left(B_{a}^{+}-b_{a}^{+}-k_{a}^{+}\right) \delta\left(b_{a}^{+}-n_{a} \cdot \hat{p}_{n_{a}}\right) \delta\left(k_{a}^{+}-n_{a} \cdot \hat{p}_{a, s}\right), \\
\delta\left(B_{b}^{+}-n_{b} \cdot \hat{p}_{b}\right) & =\int \mathrm{d} b_{b}^{+} \mathrm{d} k_{b}^{+} \delta\left(B_{b}^{+}-b_{b}^{+}-k_{b}^{+}\right) \delta\left(b_{b}^{+}-n_{b} \cdot \hat{p}_{n_{b}}\right) \delta\left(k_{b}^{+}-n_{b} \cdot \hat{p}_{b, s}\right),
\end{aligned}
$$

where $b_{a}^{\mu}, b_{b}^{\mu}$ and $k_{a}^{\mu}, k_{b}^{\mu}$ are the contributions of the collinear and soft momenta from each hemisphere respectively. We also apply the multipole expansion to the arguments of the collinear and soft fields in $x_{\mu}$ so that the collinear fields are evaluated at $x_{\mu}^{+}$, the anti-collinear fields at $x_{\mu}^{-}$and the soft fields at the origin. We rewrite the delta function constraints on the collinear fields in position space by introducing two integrations over the components $x_{a}^{-}$and $x_{b}^{-}$and insert the following identities

$$
1=e^{+i x_{b}^{-} n_{b} \cdot \hat{p}_{n_{b}} / 2} e^{-i x_{b}^{-} n_{b} \cdot \hat{p}_{n_{b}} / 2}, \quad 1=e^{-i x_{a}^{-} n_{a} \cdot \hat{p}_{n_{a}} / 2} e^{i x_{a}^{-} n_{a} \cdot \hat{p}_{n_{a}} / 2},
$$


between the bra (or ket) and the collinear (anti-collinear) fields to translate the field arguments by $x_{a}^{-} n_{a}^{\mu} / 2\left(x_{b}^{-} n_{b}^{\mu} / 2\right)$. In addition, we use the fact that the momentum operators $n_{a} \cdot \hat{p}_{n_{a}}$ and $n_{b} \cdot \hat{p}_{n_{b}}$ acting on proton states give

$$
n_{a} \cdot \hat{p}_{n_{a}}\left|P_{1}\left(P_{a}\right)\right\rangle=0, \quad n_{b} \cdot \hat{p}_{n_{b}}\left|P_{2}\left(P_{b}\right)\right\rangle=0 .
$$

We obtain

$$
\begin{aligned}
& \frac{\mathrm{d} \sigma^{g g}}{\mathrm{~d} M \mathrm{~d} y_{t \bar{t}} \mathrm{~d} \cos \theta \mathrm{d} B_{a}^{+} \mathrm{d} B_{b}^{+}}= \\
& \frac{M \beta_{t}}{32 S} \frac{1}{(2 \pi)^{4}} \frac{1}{4 d_{R}^{2}} \int \mathrm{d} k_{a}^{+} \mathrm{d} k_{b}^{+} \mathrm{d} b_{a}^{+} \mathrm{d} b_{b}^{+} \delta\left(B_{a}^{+}-b_{a}^{+}-k_{a}^{+}\right) \delta\left(B_{b}^{+}-b_{b}^{+}-k_{b}^{+}\right) \\
& \times \int \mathrm{d} \phi_{t} \int \mathrm{d} x^{+} \mathrm{d} x^{-} e^{-i\left(q^{-} x^{+}+q^{+} x^{-}\right) / 2} \int \mathrm{d} r^{\prime} \mathrm{d} t^{\prime} \mathrm{d} r \mathrm{~d} t \\
& \times \sum_{m, m^{\prime}}\left|C_{m}(r, t)\right\rangle\left\langle C_{m^{\prime}}\left(r^{\prime}, t^{\prime}\right)\right|\left\langle 0\left|\left[O_{m^{\prime}}^{h \dagger}(0)\right]^{\rho \sigma}\right| t\left(p_{3}\right) \bar{t}\left(p_{4}\right)\right\rangle\left\langle t\left(p_{3}\right) \bar{t}\left(p_{4}\right)\left|\left[O_{m}^{h}(0)\right]^{\mu \nu}\right| 0\right\rangle \\
& \times\left\langle P_{1}\left(P_{a}\right)\left|\mathcal{A}_{c \rho \perp}\left(x^{+} n_{b} / 2+t^{\prime} n_{b}\right) \delta\left(b_{a}^{+}-n_{a} \cdot \hat{p}_{n_{a}}\right) \mathcal{A}_{c \mu \perp}\left(t n_{b}\right)\right| P_{1}\left(P_{a}\right)\right\rangle \\
& \times\left\langle P_{2}\left(P_{b}\right)\left|\mathcal{A}_{\bar{c} \sigma \perp}\left(x^{-} n_{a} / 2+r^{\prime} n_{a}\right) \delta\left(b_{b}^{+}-n_{b} \cdot \hat{p}_{n_{b}}\right) \mathcal{A}_{\bar{c} \nu \perp}\left(r n_{a}\right)\right| P_{2}\left(P_{b}\right)\right\rangle \\
& \times\left\langle 0\left|\overline{\mathbf{T}}\left[\boldsymbol{O}^{s \dagger}(0)\right] \delta\left(k_{a}^{+}-n_{a} \cdot \hat{p}_{a, s}\right) \delta\left(k_{b}^{+}-n_{b} \cdot \hat{p}_{b, s}\right) \mathbf{T}\left[\boldsymbol{O}^{s}(0)\right]\right| 0\right\rangle \\
& =\frac{M \beta_{t}}{32 S} \frac{1}{(2 \pi)^{4}} \frac{1}{4 d_{R}^{2}} \int \mathrm{d} k_{a}^{+} \mathrm{d} k_{b}^{+} \mathrm{d} b_{a}^{+} \mathrm{d} b_{b}^{+} \delta\left(B_{a}^{+}-b_{a}^{+}-k_{a}^{+}\right) \delta\left(B_{b}^{+}-b_{b}^{+}-k_{b}^{+}\right) \\
& \times \int \mathrm{d} \phi_{t} \int \mathrm{d} x^{+} \mathrm{d} x^{-} e^{-i\left(q^{-} x^{+}+q^{+} x^{-}\right) / 2} \int \mathrm{d} r^{\prime} \mathrm{d} t^{\prime} \mathrm{d} r \mathrm{~d} t \\
& \times \sum_{m, m^{\prime}}\left|C_{m}(r, t)\right\rangle\left\langle C_{m^{\prime}}\left(r^{\prime}, t^{\prime}\right)\right|\left\langle 0\left|\left[O_{m^{\prime}}^{h \dagger}(0)\right]^{\rho \sigma}\right| t\left(p_{3}\right) \bar{t}\left(p_{4}\right)\right\rangle\left\langle t\left(p_{3}\right) \bar{t}\left(p_{4}\right)\left|\left[O_{m}^{h}(0)\right]^{\mu \nu}\right| 0\right\rangle \\
& \times\left(\int \frac{\mathrm{d} x_{a}^{-}}{4 \pi} e^{+i x_{a}^{-} b_{a}^{+} / 2}\left\langle P_{1}\left(P_{a}\right)\left|\mathcal{A}_{c \rho \perp}\left(x^{+} n_{b} / 2+x_{a}^{-} n_{a} / 2+t^{\prime} n_{b}\right) \mathcal{A}_{c \mu \perp}\left(\operatorname{tn}_{b}\right)\right| P_{1}\left(P_{a}\right)\right\rangle\right) \\
& \times\left(\int \frac{\mathrm{d} x_{b}^{-}}{4 \pi} e^{+i x_{b}^{-} b_{b}^{+} / 2}\left\langle P_{2}\left(P_{b}\right)\left|\mathcal{A}_{\bar{c} \sigma \perp}\left(x^{-} n_{a} / 2+x_{b}^{-} n_{b} / 2+r^{\prime} n_{a}\right) \mathcal{A}_{\bar{c} \nu \perp}\left(r n_{a}\right)\right| P_{2}\left(P_{b}\right)\right\rangle\right) \\
& \times\left(\sum_{X_{s}}\left\langle 0\left|\overline{\mathbf{T}}\left[\boldsymbol{O}^{s \dagger}(0)\right]\right| X_{s}\right\rangle\left\langle X_{s}\left|\mathbf{T}\left[\boldsymbol{O}^{s}(0)\right]\right| 0\right\rangle \delta\left(k_{a}^{+}-k_{a}^{+}\left(X_{s}\right)\right) \delta\left(k_{b}^{+}-k_{b}^{+}\left(X_{s}\right)\right)\right) .
\end{aligned}
$$

In the last line of the equation above we have also introduced the eigenvalues $k_{i}^{+}\left(X_{s}\right)$ of the projection of the momentum operator on a soft state $\left|X_{s}\right\rangle$ via $n_{i} \cdot \hat{p}_{i, s}\left|X_{s}\right\rangle=k_{i}^{+}\left(X_{s}\right)\left|X_{s}\right\rangle$. At this point we introduce an operatorial definition for the gluon beam function $B_{g / P_{1}}$ as follows

$$
\begin{aligned}
& \left\langle P_{1}\left(P_{a}\right)\left|\mathcal{A}_{c \rho \perp}^{a}\left(x^{+} \bar{n}_{a} / 2+x_{a}^{-} n_{a} / 2+t^{\prime} \bar{n}_{a}\right) \mathcal{A}_{c \mu \perp}^{b}\left(t \bar{n}_{a}\right)\right| P_{1}\left(P_{a}\right)\right\rangle= \\
& \left(-g_{\rho \mu \perp}\right) \delta^{a b}\left(\bar{n}_{a} \cdot P_{a}\right) \int_{0}^{1} \mathrm{~d} z_{a} e^{i z_{a}\left[x^{+} \bar{n}_{a} / 2+\left(t^{\prime}-t\right) \bar{n}_{a}\right] \cdot P_{a}} \int \mathrm{d} b_{a}^{+\prime} e^{-i\left(x_{a}^{-} b_{a}^{+\prime}\right) / 2} \\
& \quad \times B_{g / P_{1}}\left(z_{a}\left(\bar{n}_{a} \cdot P_{a}\right) b_{a}^{+\prime}, z_{a}, \mu\right) .
\end{aligned}
$$

Notice that the Fourier transform which appears in the equation above misses a factor $1 /(4 \pi)$ compared to the standard Fourier transform conventions in ref. [72]. We do this in 
order to normalise the tree level beam functions in the same way as in ref. [48], namely $B_{i}^{(0)}(t, z, \mu)=\delta(t) f_{i}(z, \mu)$ where the $f_{i}$ are the standard parton distribution functions. We also define the soft functions

$$
\begin{aligned}
& \mathbf{S}_{g g}\left(k_{a}^{+}, k_{b}^{+}, \beta_{t}, \theta, \mu\right)= \\
& \frac{1}{d_{R}}\left(\sum_{X_{s}}\left\langle 0\left|\overline{\mathbf{T}}\left[\boldsymbol{O}^{s \dagger}(0)\right]\right| X_{s}\right\rangle\left\langle X_{s}\left|\mathbf{T}\left[\boldsymbol{O}^{s}(0)\right]\right| 0\right\rangle \delta\left(k_{a}^{+}-k_{a}^{+}\left(X_{s}\right)\right) \delta\left(k_{b}^{+}-k_{b}^{+}\left(X_{s}\right)\right)\right),
\end{aligned}
$$

and hard functions

$$
\begin{aligned}
\mathbf{H}_{g g}^{\mu \nu \rho \sigma}\left(M, \beta_{t}, v_{3}, \mu\right)= & \frac{1}{(4 \pi)^{2}} \frac{1}{4 d_{R}} \sum_{m, m^{\prime}}\left|\tilde{C}_{m}\right\rangle\left\langle\tilde{C}_{m^{\prime}}\right| \\
& \times\left\langle 0\left|\left[O_{m^{\prime}}^{h \dagger}(0)\right]^{\rho \sigma}\right| t\left(p_{3}\right) \bar{t}\left(p_{4}\right)\right\rangle\left\langle t\left(p_{3}\right) \bar{t}\left(p_{4}\right)\left|\left[O_{m}^{h}(0)\right]^{\mu \nu}\right| 0\right\rangle,
\end{aligned}
$$

where

$$
\left|\tilde{C}_{m}\right\rangle \equiv\left|\tilde{C}_{m}\left(M, \beta_{t}, \theta, \mu\right)\right\rangle=\int \mathrm{d} r \mathrm{~d} t e^{-i z_{a} t n_{b} \cdot P_{a}} e^{-i z_{b} r n_{a} \cdot P_{b}}\left|C_{m}(r, t)\right\rangle .
$$

Since the two beam functions are proportional to $g_{\perp}^{\rho \mu}$ and $g_{\perp}^{\sigma \nu}$ and are contracted with the open Lorentz indices of the hard functions, we can define a scalar hard function as

$$
\mathbf{H}_{g g}\left(M, \beta_{t}, \theta, \mu\right)=\left(-g_{\rho \mu \perp}\right)\left(-g_{\sigma \nu \perp}\right) \mathbf{H}_{g g}^{\mu \nu \rho \sigma}\left(M, \beta_{t}, v_{3}, \mu\right) .
$$

Furthermore, we integrate over $\phi_{t}$ because the integrand is independent of this. After these substitutions and integration over $x_{a}^{-}, x_{b}^{-}$the result for the differential cross section in the gluon channel reads

$$
\begin{aligned}
& \frac{\mathrm{d} \sigma^{g g}}{\mathrm{~d} M \mathrm{~d} y_{t \bar{t}} \mathrm{~d} \cos \theta \mathrm{d} B_{a}^{+} \mathrm{d} B_{b}^{+}}= \\
& \frac{\beta_{t} M}{32 S} \frac{1}{(2 \pi)^{3}} \int \mathrm{d} k_{a}^{+} \mathrm{d} k_{b}^{+} \int \mathrm{d} b_{a}^{+} \mathrm{d} b_{b}^{+} \delta\left(B_{a}^{+}-b_{a}^{+}-k_{a}^{+}\right) \delta\left(B_{b}^{+}-b_{b}^{+}-k_{b}^{+}\right) \\
& \times S \int \mathrm{d} z_{a} \mathrm{~d} z_{b} \int \mathrm{d} x^{+} \mathrm{d} x^{-} e^{-i\left(q^{-} x^{+}+q^{+} x^{-}\right) / 2} e^{i z_{a} x^{+} n_{b} \cdot P_{a} / 2} e^{i z_{b} x^{-} n_{a} \cdot P_{b} / 2} \\
& \times \int \mathrm{d} r^{\prime} \mathrm{d} t^{\prime} \mathrm{d} r \mathrm{~d} t e^{i z_{a}\left(t^{\prime}-t\right) n_{b} \cdot P_{a}} e^{i z_{b}\left(r-r^{\prime}\right) n_{a} \cdot P_{b}} \\
& \times\left[\left(-g_{\rho \mu \perp}\right) B_{g / P_{1}}\left(z_{a}\left(n_{b} \cdot P_{a}\right) b_{a}^{+}, z_{a}, \mu\right)\left(-g_{\sigma \nu \perp}\right) B_{g / P_{2}}\left(z_{b}\left(n_{a} \cdot P_{b}\right) b_{b}^{+}, z_{b}, \mu\right)\right] \\
& \times \frac{1}{4 d_{R}} \operatorname{Tr}\left\{\sum_{m, m^{\prime}}\left|C_{m}(r, t)\right\rangle\left\langle C_{m^{\prime}}\left(r^{\prime}, t^{\prime}\right)\right|\left\langle 0\left|\left[O_{m^{\prime}}^{h \dagger}(0)\right]^{\rho \sigma}\right| t\left(p_{3}\right) \bar{t}\left(p_{4}\right)\right\rangle\left\langle t\left(p_{3}\right) \bar{t}\left(p_{4}\right)\left|\left[O_{m}^{h}(0)\right]^{\mu \nu}\right| 0\right\rangle\right. \\
& \left.\quad \mathbf{S}_{g g}\left(k_{a}^{+}, k_{b}^{+}, \beta_{t}, \theta, \mu\right)\right\} \\
& =\frac{\pi \beta_{t} M}{} \quad S \mathrm{~d} k_{a}^{+} \mathrm{d} k_{b}^{+} \int \mathrm{d} b_{a}^{+} \mathrm{d} b_{b}^{+} \delta\left(B_{a}^{+}-b_{a}^{+}-k_{a}^{+}\right) \delta\left(B_{b}^{+}-b_{b}^{+}-k_{b}^{+}\right) \\
& \quad \times \int \mathrm{d} z_{a} \mathrm{~d} z_{b} \delta\left(z_{a}-\frac{q^{-}}{n_{b} \cdot P_{a}}\right) \delta\left(z_{b}-\frac{q^{+}}{n_{a} \cdot P_{b}}\right) \\
& \quad \times\left[B_{g / P_{1}}\left(z_{a}\left(n_{b} \cdot P_{a}\right) b_{a}^{+}, z_{a}, \mu\right) B_{g / P_{2}}\left(z_{b}\left(n_{a} \cdot P_{b}\right) b_{b}^{+}, z_{b}, \mu\right)\right] \\
& \quad \times \operatorname{Tr}\left\{\mathbf{H}_{g g}\left(M, \beta_{t}, \theta, \mu\right) \mathbf{S}_{g g}\left(k_{a}^{+}, k_{b}^{+}, \beta_{t}, \theta, \mu\right)\right\}
\end{aligned}
$$




$$
\begin{aligned}
= & \frac{\pi \beta_{t} M}{S} \int \mathrm{d} k_{a}^{+} \mathrm{d} k_{b}^{+} \operatorname{Tr}\left\{\mathbf{H}_{g g}\left(M, \beta_{t}, \theta, \mu\right) \mathbf{S}_{g g}\left(k_{a}^{+}, k_{b}^{+}, \beta_{t}, \theta, \mu\right)\right\} \\
& \times\left[B_{g / P_{1}}\left(q^{-}\left(B_{a}^{+}-k_{a}^{+}\right), q^{-} / n_{b} \cdot P_{a}, \mu\right) B_{g / P_{2}}\left(q^{+}\left(B_{b}^{+}-k_{b}^{+}\right), q^{+} / n_{a} \cdot P_{b}, \mu\right)\right],
\end{aligned}
$$

where $q^{\mp}=M e^{ \pm y_{t \bar{t}}}$ and the Kronecker deltas with colour indices arising from the beam functions produce a colour trace over the product of the hard and soft functions.

We now wish to define a variable to constrain both hemispheres simultaneously. When the rapidity of the $t \bar{t}$ system is zero, the hemispheres are equal in size and a good choice is

$$
\widehat{B}=\frac{B_{a}^{+}+B_{b}^{+}}{M} .
$$

Integrating over $B_{a}^{+}$and $B_{b}^{+}$we obtain

$$
\begin{aligned}
& \frac{\mathrm{d} \sigma^{g g}}{\mathrm{~d} M \mathrm{~d} y_{t \bar{t}} \mathrm{~d} \cos \theta \mathrm{d} \widehat{B}}=\frac{\pi \beta_{t} M}{S} \int \mathrm{d} k_{a}^{+} \mathrm{d} k_{b}^{+} \mathrm{d} B_{a}^{+} \mathrm{d} B_{b}^{+} \delta\left(M \widehat{B}-B_{a}^{+}-B_{b}^{+}\right) \\
& \quad \times\left[B_{g / P_{1}}\left(q^{-}\left(B_{a}^{+}-k_{a}^{+}\right), q^{-} / n_{b} \cdot P_{a}, \mu\right) B_{g / P_{2}}\left(q^{+}\left(B_{b}^{+}-k_{b}^{+}\right), q^{+} / n_{a} \cdot P_{b}, \mu\right)\right] \\
& \quad \times \operatorname{Tr}\left\{\mathbf{H}_{g g}\left(M, \beta_{t}, \theta, \mu\right) \mathbf{S}_{g g}\left(k_{a}^{+}, k_{b}^{+}, \beta_{t}, \theta, \mu\right)\right\} \\
& =\frac{\pi \beta_{t}}{M S} \int \mathrm{d} t_{a} \mathrm{~d} t_{b}\left[B_{g / P_{1}}\left(t_{a}, z_{a}, \mu\right) B_{g / P_{2}}\left(t_{b}, z_{b}, \mu\right)\right] \\
& \quad \times \operatorname{Tr}\left\{\mathbf{H}_{g g}\left(M, \beta_{t}, \theta, \mu\right) \mathbf{S}_{B, g g}\left(M \widehat{B}-\frac{t_{a}}{q^{-}}-\frac{t_{b}}{q^{+}}, \beta_{t}, \theta, \mu\right)\right\},
\end{aligned}
$$

where we have used that $B_{a, b}^{+}=k_{a, b}^{+}+b_{a, b}^{+}, t_{a}=q^{-} b_{a}^{+}$and $t_{b}=q^{+} b_{b}^{+}$, and have defined the soft function as

$$
\mathbf{S}_{B, g g}\left(l^{+}, \beta_{t}, \theta, \mu\right)=\int \mathrm{d} k_{a}^{+} \mathrm{d} k_{b}^{+} \mathbf{S}_{g g}\left(k_{a}^{+}, k_{b}^{+}, \beta_{t}, \theta, \mu\right) \delta\left(l^{+}-k_{a}^{+}-k_{b}^{+}\right) .
$$

Boosting the $t \bar{t}$ system to a generic rapidity $y_{t \bar{t}}$ will, however, alter not only the distribution of the QCD radiation $B_{i}^{+}\left(y_{t \bar{t}}\right)$ in the hemisphere $i=\{a, b\}$ but also change the definitions of the hemispheres themselves. Defining rapidity-dependent hemispheres $a$ and $b$ for $y>y_{t \bar{t}}$ and $y<y_{t \bar{t}}$, we introduce the boost-invariant combination

$$
\tau_{B}=\frac{q^{-} B_{a}^{+}\left(y_{t \bar{t}}\right)+q^{+} B_{b}^{+}\left(y_{t \bar{t}}\right)}{M^{2}} .
$$

In the partonic centre-of-mass frame, the definition of $\tau_{B}$ in eq. (A.38) coincides with the definition of $\widehat{B}$ in eq. (A.35) and one can replace $\widehat{B}$ in eq. (A.36) with $\tau_{B}$. Boosting to a generic frame at rapidity $y_{t \bar{t}} \neq 0$, the (invariant) beam functions are unaffected: the soft function, on the other hand, is boost-invariant up to the hemisphere definition which defines its arguments $k_{a, b}^{+}$. It can easily be shown [48] that the simultaneous transformation of the soft function and its arguments results in the soft function for $\tau_{B}$ being the same as for $\widehat{B}$ we can therefore write

$$
\begin{aligned}
\frac{\mathrm{d} \sigma^{g g}}{\mathrm{~d} M \mathrm{~d} y_{t \bar{t}} \mathrm{~d} \cos \theta \mathrm{d} \tau_{B}}= & \frac{\pi \beta_{t}}{M S} \int \mathrm{d} t_{a} \mathrm{~d} t_{b}\left[B_{g / P_{1}}\left(t_{a}, z_{a}, \mu\right) B_{g / P_{2}}\left(t_{b}, z_{b}, \mu\right)\right] \\
& \times \operatorname{Tr}\left\{\mathbf{H}_{g g}\left(M, \beta_{t}, \theta, \mu\right) \mathbf{S}_{B, g g}\left(M \tau_{B}-\frac{t_{a}+t_{b}}{M}, \beta_{t}, \theta, \mu\right)\right\} .
\end{aligned}
$$


The derivation of the factorisation formula for the quark channel follows similarly to the gluon case. The main difference involves the operatorial definition of the quark beam function $B_{q / P_{1}}$ which is given by

$$
\begin{aligned}
& \left\langle P_{1}\left(P_{a}\right)\left|\bar{\chi}_{c}\left(x^{+} \bar{n}_{a} / 2+x_{a}^{-} n_{a} / 2+t^{\prime} \bar{n}_{a}\right) \frac{\not h_{b}}{2} \chi_{c}\left(t \bar{n}_{a}\right)\right| P_{1}\left(P_{a}\right)\right\rangle= \\
& \left(\bar{n}_{a} \cdot P_{a}\right)^{2} \int_{0}^{1} \mathrm{~d} z_{a} z_{a} e^{i z_{a}\left[x^{+} \bar{n}_{a} / 2+\left(t^{\prime}-t\right) \bar{n}_{a}\right] \cdot P_{a}} \int \mathrm{d} b_{a}^{+} e^{-i\left(x_{a}^{-} b_{a}^{+}\right) / 2} \\
& \times B_{q / P_{1}}\left(z_{a}\left(\bar{n}_{a} \cdot P_{a}\right) b_{a}^{+}, z_{a}, \mu\right) .
\end{aligned}
$$

\section{B One-loop soft integrals}

In this appendix we collect the integrals that need to be evaluated for the one-loop soft functions. We have analytically calculated the pole structure of all integrals. The terms at finite order in the regulator $\epsilon$, however, resisted our attempts at an analytic calculation, leaving several finite one-fold integrals to be performed numerically. ${ }^{8}$

We parameterise the momenta of the soft parton and of the top and anti-top quarks as

$$
\begin{aligned}
k & =k_{0}(1, \ldots, \sin \chi \sin \phi, \sin \chi \cos \phi, \cos \chi), \\
v_{3} & =\frac{1}{\sqrt{1-\beta_{t}^{2}}}\left(1, \ldots, 0, \beta_{t} \sin \theta, \beta_{t} \cos \theta\right), \\
v_{4} & =\frac{1}{\sqrt{1-\beta_{t}^{2}}}\left(1, \ldots, 0,-\beta_{t} \sin \theta,-\beta_{t} \cos \theta\right),
\end{aligned}
$$

respectively. We have $\beta_{t}=\sqrt{1-4 m_{t}^{2} / M^{2}}$ and $M$ is the invariant mass of the $t \bar{t}$ pair. The integration measure is

$$
\mathrm{d}^{d} k \delta\left(k^{2}\right) \Theta\left(k^{0}\right)=\Omega_{d-4} \frac{k_{0}^{1-2 \epsilon}}{2} \mathrm{~d} k_{0} \sin ^{1-2 \epsilon} \chi \mathrm{d} \chi \sin ^{-2 \epsilon} \phi \mathrm{d} \phi
$$

and the volume of the $d-4=-2 \epsilon$ space is given by

$$
\Omega_{d-4}=\frac{2 \pi^{1 / 2-\epsilon}}{\Gamma\left(\frac{1}{2}-\epsilon\right)}=\frac{2^{1-2 \epsilon} \pi^{-\epsilon} \Gamma(1-\epsilon)}{\Gamma(1-2 \epsilon)} .
$$

We also define the variable $z=\cos \chi$. In the following we collect the relevant integrals, separated according to the configuration of massless and massive legs and their directions with respect to the hemispheres.

\footnotetext{
${ }^{8}$ Analytic results for the finite terms in $\epsilon$ of similar integrals have been presented in refs. [90, 91]. However, in our case the differing phase space constraints mean that we are unable to make use of these results; specifically, since we integrate over each hemisphere separately, we introduce a step function in the cosine of the angle $\chi$. This is absent in the aforementioned works.
} 


\section{B.1 Heavy-heavy integrals, same leg}

$$
\begin{aligned}
& I_{v_{3} v_{3}}=\int \mathrm{d}^{d} k \delta\left(k^{2}\right) \Theta\left(k_{0}\right) \frac{v_{3} \cdot v_{3}}{\left(v_{3} \cdot k\right)^{2}} \delta\left(l^{+}-k \cdot n_{a}\right) \Theta\left(k \cdot n_{b}-k \cdot n_{a}\right) \\
& =\frac{1}{2} \Omega_{d-4} \int \mathrm{d} k_{0} \mathrm{~d} \chi \mathrm{d} \phi k_{0}^{1-2 \epsilon} \sin ^{1-2 \epsilon} \chi \sin ^{-2 \epsilon} \phi \frac{1-\beta_{t}^{2}}{k_{0}^{2}} \\
& \times\left(1-\beta_{t} \sin \chi \cos \phi \sin \theta-\beta_{t} \cos \chi \cos \theta\right)^{-2} \delta\left(l^{+}-k_{0}(1-\cos \chi)\right) \Theta(\cos \chi) \\
& =\frac{1}{2} \Omega_{d-4} \int_{0}^{\pi} \mathrm{d} \phi \int_{0}^{1} \mathrm{~d} z\left(l^{+}\right)^{-1-2 \epsilon}\left(1-\beta_{t}^{2}\right)\left(\frac{1-z}{1+z}\right)^{\epsilon} \sin ^{-2 \epsilon} \phi \\
& \times\left(1-\beta_{t} \sqrt{1-z^{2}} \cos \phi \sin \theta-\beta_{t} z \cos \theta\right)^{-2} \\
& =\frac{\pi}{2} \Omega_{d-4}\left(l^{+}\right)^{-1-2 \epsilon}\left(1+\frac{\beta_{t} \cos \theta}{\sqrt{1-\beta_{t}^{2} \sin ^{2} \theta}}+\mathcal{O}(\epsilon)\right) \\
& I_{v_{4} v_{4}}=\int \mathrm{d}^{d} k \delta\left(k^{2}\right) \Theta\left(k_{0}\right) \frac{v_{4} \cdot v_{4}}{\left(v_{4} \cdot k\right)^{2}} \delta\left(l^{+}-k \cdot n_{a}\right) \Theta\left(k \cdot n_{b}-k \cdot n_{a}\right) \\
& =\frac{1}{2} \Omega_{d-4} \int \mathrm{d} k_{0} \mathrm{~d} \chi \mathrm{d} \phi k_{0}^{1-2 \epsilon} \sin ^{1-2 \epsilon} \chi \sin ^{-2 \epsilon} \phi \frac{1-\beta_{t}^{2}}{k_{0}^{2}} \\
& \times\left(1+\beta_{t} \sin \chi \cos \phi \sin \theta+\beta_{t} \cos \chi \cos \theta\right)^{-2} \delta\left(l^{+}-k_{0}(1-\cos \chi)\right) \Theta(\cos \chi) \\
& =\frac{1}{2} \Omega_{d-4} \int_{0}^{\pi} \mathrm{d} \phi \int_{0}^{1} \mathrm{~d} z\left(l^{+}\right)^{-1-2 \epsilon}\left(1-\beta_{t}^{2}\right)\left(\frac{1-z}{1+z}\right)^{\epsilon} \sin ^{-2 \epsilon} \phi \\
& \times\left(1+\beta_{t} \sqrt{1-z^{2}} \cos \phi \sin \theta+\beta_{t} z \cos \theta\right)^{-2} \\
& =\frac{\pi}{2} \Omega_{d-4}\left(l^{+}\right)^{-1-2 \epsilon}\left(1-\frac{\beta_{t} \cos \theta}{\sqrt{1-\beta_{t}^{2} \sin ^{2} \theta}}+\mathcal{O}(\epsilon)\right)
\end{aligned}
$$

\section{B.2 Heavy-heavy integrals, opposite leg}

$$
\begin{aligned}
& I_{v_{3} v_{4}}= \int \mathrm{d}^{d} k \delta\left(k^{2}\right) \Theta\left(k_{0}\right) \frac{v_{3} \cdot v_{4}}{\left(v_{3} \cdot k\right)\left(v_{4} \cdot k\right)} \delta\left(l^{+}-k \cdot n_{a}\right) \Theta\left(k \cdot n_{b}-k \cdot n_{a}\right) \\
&= \frac{1}{2} \Omega_{d-4} \int \mathrm{d} k_{0} \mathrm{~d} \chi \mathrm{d} \phi k_{0}^{1-2 \epsilon} \sin ^{1-2 \epsilon} \chi \sin ^{-2 \epsilon} \phi \frac{1+\beta_{t}^{2}}{k_{0}^{2}} \\
& \quad \times\left[1-\beta_{t}^{2}\left(\sin \chi \cos \phi \sin \theta+\beta_{t} \cos \chi \cos \theta\right)^{2}\right]^{-1} \delta\left(l^{+}-k_{0}(1-\cos \chi)\right) \Theta(\cos \chi) \\
&=\frac{1}{2} \Omega_{d-4} \int_{0}^{\pi} \mathrm{d} \phi \int_{0}^{1} \mathrm{~d} z\left(l^{+}\right)^{-1-2 \epsilon}\left(1+\beta_{t}^{2}\right)\left(\frac{1-z}{1+z}\right)^{\epsilon} \sin ^{-2 \epsilon} \phi \\
& \quad \times\left[1-\beta_{t}^{2}\left(\sqrt{1-z^{2}} \cos \phi \sin \theta+\beta_{t} z \cos \theta\right)^{2}\right]^{-1} \\
&=\frac{\pi}{2} \Omega_{d-4}\left(l^{+}\right)^{-1-2 \epsilon} \frac{1+\beta_{t}^{2}}{2 \beta_{t}}\left[\ln \left(\frac{1+\beta_{t}}{1-\beta_{t}}\right)+\mathcal{O}(\epsilon)\right] \\
& \quad I_{v_{4} v_{3}}=I_{v_{3} v_{4}}
\end{aligned}
$$




\section{B.3 Heavy-light integrals, same hemisphere}

$$
\begin{aligned}
I_{n_{a} v_{3}}= & \int \mathrm{d}^{d} k \delta\left(k^{2}\right) \Theta\left(k_{0}\right) \frac{n_{a} \cdot v_{3}}{\left(n_{a} \cdot k\right)\left(v_{3} \cdot k\right)} \delta\left(l^{+}-k \cdot n_{a}\right) \Theta\left(k \cdot n_{b}-k \cdot n_{a}\right) \\
= & \frac{1}{2} \Omega_{d-4} \int \mathrm{d} k_{0} \mathrm{~d} \chi \mathrm{d} \phi k_{0}^{1-2 \epsilon} \sin ^{1-2 \epsilon} \chi \sin ^{-2 \epsilon} \phi \frac{1-\beta_{t} \cos \theta}{k_{0}^{2}(1-\cos \chi)} \\
& \quad \times\left(1-\beta_{t} \sin \chi \cos \phi \sin \theta-\beta_{t} \cos \chi \cos \theta\right)^{-1} \delta\left(l^{+}-k_{0}(1-\cos \chi)\right) \Theta(\cos \chi) \\
= & \frac{1}{2} \Omega_{d-4} \int_{0}^{\pi} \mathrm{d} \phi \int_{0}^{1} \mathrm{~d} z\left(l^{+}\right)^{-1-2 \epsilon}\left(1-\beta_{t} \cos \theta\right)(1-z)^{-1+\epsilon}(1+z)^{-\epsilon} \sin ^{-2 \epsilon} \phi
\end{aligned}
$$

\section{B.4 Heavy-light integrals, opposite hemisphere}

$$
\begin{aligned}
I_{n_{a} v_{4}}= & \int \mathrm{d}^{d} k \delta\left(k^{2}\right) \Theta\left(k_{0}\right) \frac{n_{a} \cdot v_{4}}{\left(n_{a} \cdot k\right)\left(v_{4} \cdot k\right)} \delta\left(l^{+}-k \cdot n_{a}\right) \Theta\left(k \cdot n_{b}-k \cdot n_{a}\right) \\
= & \frac{1}{2} \Omega_{d-4} \int \mathrm{d} k_{0} \mathrm{~d} \chi \mathrm{d} \phi k_{0}^{1-2 \epsilon} \sin ^{1-2 \epsilon} \chi \sin ^{-2 \epsilon} \phi \frac{1+\beta_{t} \cos \theta}{k_{0}^{2}(1-\cos \chi)} \\
& \quad \times\left(1+\beta_{t} \sin \chi \cos \phi \sin \theta+\beta_{t} \cos \chi \cos \theta\right)^{-1} \delta\left(l^{+}-k_{0}(1-\cos \chi)\right) \Theta(\cos \chi) \\
= & \frac{1}{2} \Omega_{d-4} \int_{0}^{\pi} \mathrm{d} \phi \int_{0}^{1} \mathrm{~d} z\left(l^{+}\right)^{-1-2 \epsilon}\left(1+\beta_{t} \cos \theta\right)(1-z)^{-1+\epsilon}(1+z)^{-\epsilon} \sin ^{-2 \epsilon} \phi \\
& \quad \times\left(1+\beta_{t} \sqrt{1-z^{2}} \cos \phi \sin \theta+\beta_{t} z \cos \theta\right)^{-1} \\
= & \frac{\pi}{2} \Omega_{d-4}\left(l^{+}\right)^{-1-2 \epsilon}\left\{\frac{1}{\epsilon}+3 \ln (2)+2 \ln \left(1+\beta_{t} \cos \theta\right)\right. \\
& \left.-\ln \left[\left(1+\beta_{t} \cos \theta\right)\left(2+\sqrt{4-2 \beta_{t}^{2}+2 \beta_{t}^{2} \cos 2 \theta}\right)-2 \beta_{t}^{2} \sin ^{2} \theta\right]+\mathcal{O}(\epsilon)\right\}
\end{aligned}
$$




$$
\begin{aligned}
I_{n_{b} v_{3}}= & \int \mathrm{d}^{d} k \delta\left(k^{2}\right) \Theta\left(k_{0}\right) \frac{n_{b} \cdot v_{3}}{\left(n_{b} \cdot k\right)\left(v_{3} \cdot k\right)} \delta\left(l^{+}-k \cdot n_{a}\right) \Theta\left(k \cdot n_{b}-k \cdot n_{a}\right) \\
= & \frac{1}{2} \Omega_{d-4} \int \mathrm{d} k_{0} \mathrm{~d} \chi \mathrm{d} \phi k_{0}^{1-2 \epsilon} \sin ^{1-2 \epsilon} \chi \sin ^{-2 \epsilon} \phi \frac{1+\beta_{t} \cos \theta}{k_{0}^{2}(1+\cos \chi)} \\
& \quad \times\left(1-\beta_{t} \sin \chi \cos \phi \sin \theta-\beta_{t} \cos \chi \cos \theta\right)^{-1} \delta\left(l^{+}-k_{0}(1-\cos \chi)\right) \Theta(\cos \chi) \\
= & \frac{1}{2} \Omega_{d-4} \int_{0}^{\pi} \mathrm{d} \phi \int_{0}^{1} \mathrm{~d} z\left(l^{+}\right)^{-1-2 \epsilon}\left(1+\beta_{t} \cos \theta\right)(1+z)^{-1-\epsilon}(1-z)^{\epsilon} \sin ^{-2 \epsilon} \phi \\
& \quad \times\left(1-\beta_{t} \sqrt{1-z^{2}} \cos \phi \sin \theta-\beta_{t} z \cos \theta\right)^{-1} \\
= & \frac{\pi}{2} \Omega_{d-4}\left(l^{+}\right)^{-1-2 \epsilon}\left\{-\ln (2)-\ln \left(1-\beta_{t}^{2}\right)\right. \\
& \left.+\ln \left[\left(1+\beta_{t} \cos \theta\right)\left(2+\sqrt{4-2 \beta_{t}^{2}+2 \beta_{t}^{2} \cos 2 \theta}\right)-2 \beta_{t}^{2} \sin ^{2} \theta\right]+\mathcal{O}(\epsilon)\right\}
\end{aligned}
$$

Open Access. This article is distributed under the terms of the Creative Commons Attribution License (CC-BY 4.0), which permits any use, distribution and reproduction in any medium, provided the original author(s) and source are credited.

\section{References}

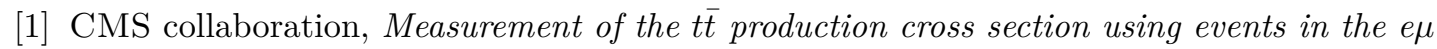
final state in pp collisions at $\sqrt{s}=13$ TeV, Eur. Phys. J. C 77 (2017) 172 [arXiv: 1611.04040] [INSPIRE].

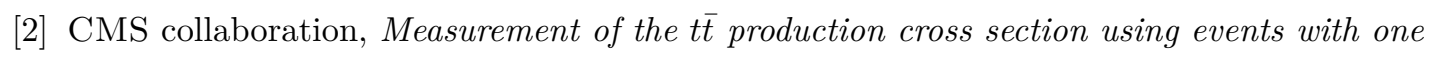
lepton and at least one jet in pp collisions at $\sqrt{s}=13 \mathrm{TeV}$, JHEP 09 (2017) 051 [arXiv: 1701.06228] [INSPIRE].

[3] CMS collaboration, Measurement of normalized differential $\mathrm{t} \overline{\mathrm{t}}$ cross sections in the dilepton channel from pp collisions at $\sqrt{s}=13 \mathrm{TeV}$, JHEP 04 (2018) 060 [arXiv:1708.07638] [INSPIRE].

[4] CMS collaboration, Measurements of t $\overline{\mathrm{t}}$ differential cross sections in proton-proton collisions at $\sqrt{s}=13 \mathrm{TeV}$ using events containing two leptons, JHEP 02 (2019) 149 [arXiv: 1811.06625] [INSPIRE].

[5] CMS collaboration, Measurements of differential cross sections of top quark pair production as a function of kinematic event variables in proton-proton collisions at $\sqrt{s}=13 \mathrm{TeV}$, JHEP 06 (2018) 002 [arXiv: 1803.03991] [INSPIRE].

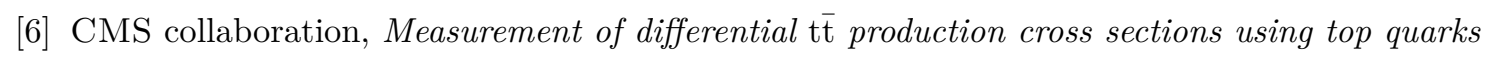
at large transverse momenta in pp collisions at $\sqrt{s}=13 \mathrm{TeV}$, Phys. Rev. D 103 (2021) 052008 [arXiv: 2008.07860] [INSPIRE].

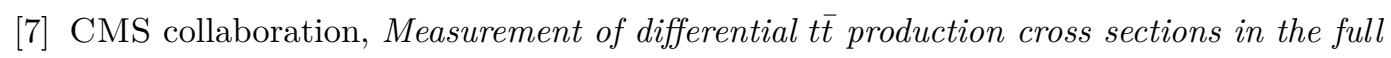
kinematic range using lepton + jets events from proton-proton collisions at $\sqrt{s}=13 \mathrm{TeV}$, Phys. Rev. D 104 (2021) 092013 [arXiv:2108. 02803] [INSPIRE].

[8] ATLAS collaboration, Measurements of top-quark pair differential and double-differential cross-sections in the $\ell+$ jets channel with pp collisions at $\sqrt{s}=13 \mathrm{TeV}$ using the ATLAS detector, Eur. Phys. J. C 79 (2019) 1028 [Erratum ibid. 80 (2020) 1092] [arXiv:1908. 07305] [INSPIRE]. 


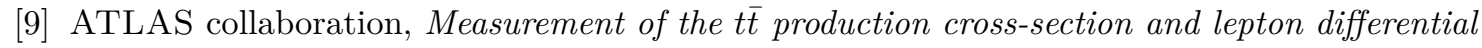
distributions in e $\mu$ dilepton events from pp collisions at $\sqrt{s}=13 \mathrm{TeV}$ with the ATLAS detector, Eur. Phys. J. C 80 (2020) 528 [arXiv:1910.08819] [InSPIRE].

[10] ATLAS collaboration, Measurement of the $t \bar{t}$ production cross-section in the lepton + jets channel at $\sqrt{s}=13 \mathrm{TeV}$ with the ATLAS experiment, Phys. Lett. B 810 (2020) 135797 [arXiv:2006.13076] [INSPIRE].

[11] ATLAS collaboration, Measurements of top-quark pair single- and double-differential cross-sections in the all-hadronic channel in pp collisions at $\sqrt{s}=13 \mathrm{TeV}$ using the ATLAS detector, JHEP 01 (2021) 033 [arXiv:2006.09274] [INSPIRE].

[12] T. Klijnsma, S. Bethke, G. Dissertori and G.P. Salam, Determination of the strong coupling constant $\alpha_{s}\left(m_{Z}\right)$ from measurements of the total cross section for top-antitop quark production, Eur. Phys. J. C 77 (2017) 778 [arXiv: 1708.07495] [InSPIRE].

[13] A.M. Cooper-Sarkar, M. Czakon, M.A. Lim, A. Mitov and A.S. Papanastasiou, Simultaneous

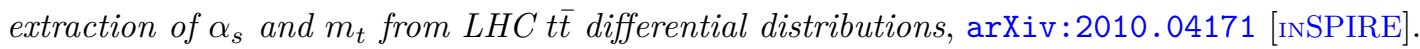

[14] P. Bärnreuther, M. Czakon and A. Mitov, Percent Level Precision Physics at the Tevatron: First Genuine NNLO QCD Corrections to $q \bar{q} \rightarrow t \bar{t}+X$, Phys. Rev. Lett. 109 (2012) 132001 [arXiv: 1204.5201] [INSPIRE].

[15] M. Czakon and A. Mitov, NNLO corrections to top pair production at hadron colliders: the quark-gluon reaction, JHEP 01 (2013) 080 [arXiv:1210.6832] [INSPIRE].

[16] M. Czakon, P. Fiedler and A. Mitov, Total Top-Quark Pair-Production Cross Section at Hadron Colliders Through $O\left(\alpha_{S}^{4}\right)$, Phys. Rev. Lett. 110 (2013) 252004 [arXiv:1303.6254] [INSPIRE].

[17] M. Czakon, D. Heymes and A. Mitov, High-precision differential predictions for top-quark pairs at the LHC, Phys. Rev. Lett. 116 (2016) 082003 [arXiv:1511.00549] [InSPIRE].

[18] M. Czakon, D. Heymes and A. Mitov, Dynamical scales for multi-TeV top-pair production at the LHC, JHEP 04 (2017) 071 [arXiv: 1606.03350] [INSPIRE].

[19] M. Czakon, P. Fiedler, D. Heymes and A. Mitov, NNLO QCD predictions for fully-differential top-quark pair production at the Tevatron, JHEP 05 (2016) 034 [arXiv:1601.05375] [INSPIRE].

[20] M. Czakon, D. Heymes and A. Mitov, fastNLO tables for NNLO top-quark pair differential distributions, arXiv:1704.08551 [INSPIRE].

[21] S. Catani, S. Devoto, M. Grazzini, S. Kallweit, J. Mazzitelli and H. Sargsyan, Top-quark pair hadroproduction at next-to-next-to-leading order in QCD, Phys. Rev. D 99 (2019) 051501 [arXiv: 1901.04005] [INSPIRE].

[22] S. Catani, S. Devoto, M. Grazzini, S. Kallweit and J. Mazzitelli, Top-quark pair production at the LHC: Fully differential QCD predictions at NNLO, JHEP 07 (2019) 100 [arXiv: 1906.06535] [INSPIRE].

[23] S. Catani, S. Devoto, M. Grazzini, S. Kallweit and J. Mazzitelli, Top-quark pair hadroproduction at NNLO: differential predictions with the $\overline{M S}$ mass, JHEP 08 (2020) 027 [arXiv : 2005.00557] [INSPIRE].

[24] A. Behring, M. Czakon, A. Mitov, A.S. Papanastasiou and R. Poncelet, Higher order corrections to spin correlations in top quark pair production at the LHC, Phys. Rev. Lett. 123 (2019) 082001 [arXiv:1901.05407] [INSPIRE]. 
[25] M. Czakon, A. Mitov and R. Poncelet, NNLO QCD corrections to leptonic observables in top-quark pair production and decay, JHEP 05 (2021) 212 [arXiv:2008.11133] [INSPIRE].

[26] W. Bernreuther, M. Fücker and Z.G. Si, Mixed QCD and weak corrections to top quark pair production at hadron colliders, Phys. Lett. B 633 (2006) 54 [Erratum ibid. 644 (2007) 386] [hep-ph/0508091] [INSPIRE].

[27] J.H. Kühn, A. Scharf and P. Uwer, Electroweak effects in top-quark pair production at hadron colliders, Eur. Phys. J. C 51 (2007) 37 [hep-ph/0610335] [INSPIRE].

[28] A. Denner and M. Pellen, NLO electroweak corrections to off-shell top-antitop production with leptonic decays at the LHC, JHEP 08 (2016) 155 [arXiv:1607.05571] [INSPIRE].

[29] M. Czakon, D. Heymes, A. Mitov, D. Pagani, I. Tsinikos and M. Zaro, Top-pair production at the LHC through NNLO QCD and NLO EW, JHEP 10 (2017) 186 [arXiv:1705.04105] [INSPIRE].

[30] A. Ferroglia, M. Neubert, B.D. Pecjak and L.L. Yang, Two-loop divergences of scattering amplitudes with massive partons, Phys. Rev. Lett. 103 (2009) 201601 [arXiv:0907.4791] [INSPIRE].

[31] A. Ferroglia, M. Neubert, B.D. Pecjak and L.L. Yang, Two-loop divergences of massive scattering amplitudes in non-abelian gauge theories, JHEP 11 (2009) 062 [arXiv:0908.3676] [INSPIRE].

[32] V. Ahrens, A. Ferroglia, M. Neubert, B.D. Pecjak and L.L. Yang, Renormalization-Group Improved Predictions for Top-Quark Pair Production at Hadron Colliders, JHEP 09 (2010) 097 [arXiv: 1003.5827] [INSPIRE].

[33] V. Ahrens, A. Ferroglia, M. Neubert, B.D. Pecjak and L.-L. Yang, RG-improved single-particle inclusive cross sections and forward-backward asymmetry in $t \bar{t}$ production at hadron colliders, JHEP 09 (2011) 070 [arXiv:1103.0550] [INSPIRE].

[34] A. Broggio, A.S. Papanastasiou and A. Signer, Renormalization-group improved fully differential cross sections for top pair production, JHEP 10 (2014) 098 [arXiv:1407.2532] [INSPIRE].

[35] J. Gao and A.S. Papanastasiou, Top-quark pair-production and decay at high precision, Phys. Rev. D 96 (2017) 051501 [arXiv:1705.08903] [INSPIRE].

[36] B.D. Pecjak, D.J. Scott, X. Wang and L.L. Yang, Resummed differential cross sections for top-quark pairs at the LHC, Phys. Rev. Lett. 116 (2016) 202001 [arXiv:1601.07020] [INSPIRE].

[37] M. Czakon et al., Resummation for (boosted) top-quark pair production at $N N L O+N N L L$ ' in QCD, JHEP 05 (2018) 149 [arXiv: 1803.07623] [INSPIRE].

[38] H.T. Li, C.S. Li, D.Y. Shao, L.L. Yang and H.X. Zhu, Top quark pair production at small transverse momentum in hadronic collisions, Phys. Rev. D 88 (2013) 074004 [arXiv: 1307.2464] [INSPIRE].

[39] S. Catani, M. Grazzini and H. Sargsyan, Transverse-momentum resummation for top-quark pair production at the LHC, JHEP 11 (2018) 061 [arXiv:1806.01601] [INSPIRE].

[40] J. Mazzitelli, P.F. Monni, P. Nason, E. Re, M. Wiesemann and G. Zanderighi, Next-to-Next-to-Leading Order Event Generation for Top-Quark Pair Production, Phys. Rev. Lett. 127 (2021) 062001 [arXiv: 2012.14267] [INSPIRE]. 
[41] P.F. Monni, P. Nason, E. Re, M. Wiesemann and G. Zanderighi, MiNNLOPS: a new method to match NNLO QCD to parton showers, JHEP 05 (2020) 143 [arXiv: 1908.06987] [INSPIRE].

[42] S. Frixione, P. Nason and G. Ridolfi, A Positive-weight next-to-leading-order Monte Carlo for heavy flavour hadroproduction, JHEP 09 (2007) 126 [arXiv:0707.3088] [INSPIRE].

[43] S. Alioli, S.-O. Moch and P. Uwer, Hadronic top-quark pair-production with one jet and parton showering, JHEP 01 (2012) 137 [arXiv:1110.5251] [INSPIRE].

[44] S. Alioli, C.W. Bauer, C. Berggren, F.J. Tackmann and J.R. Walsh, Drell-Yan production at NNLL'+NNLO matched to parton showers, Phys. Rev. D 92 (2015) 094020 [arXiv: 1508.01475] [INSPIRE].

[45] S. Alioli et al., Matching NNLO predictions to parton showers using N3LL color-singlet transverse momentum resummation in geneva, Phys. Rev. D 104 (2021) 094020 [arXiv:2102.08390] [INSPIRE].

[46] R. Angeles-Martinez, M. Czakon and S. Sapeta, NNLO soft function for top quark pair production at small transverse momentum, JHEP 10 (2018) 201 [arXiv:1809.01459] [INSPIRE].

[47] I.W. Stewart, F.J. Tackmann and W.J. Waalewijn, N-Jettiness: An Inclusive Event Shape to Veto Jets, Phys. Rev. Lett. 105 (2010) 092002 [arXiv:1004.2489] [INSPIRE].

[48] I.W. Stewart, F.J. Tackmann and W.J. Waalewijn, Factorization at the LHC: From PDFs to Initial State Jets, Phys. Rev. D 81 (2010) 094035 [arXiv:0910.0467] [InSPIRE].

[49] S. Alioli, A. Broggio, S. Kallweit, M.A. Lim and L. Rottoli, Higgsstrahlung at NNLL'+NNLO matched to parton showers in GENEVA, Phys. Rev. D 100 (2019) 096016 [arXiv: 1909.02026] [INSPIRE].

[50] S. Alioli et al., Resummed predictions for hadronic Higgs boson decays, JHEP 04 (2021) 254 [arXiv:2009.13533] [INSPIRE].

[51] S. Alioli et al., Precise predictions for photon pair production matched to parton showers in GENEVA, JHEP 04 (2021) 041 [arXiv: 2010.10498] [INSPIRE].

[52] S. Alioli et al., Next-to-next-to-leading order event generation for $Z$ boson pair production matched to parton shower, Phys. Lett. B 818 (2021) 136380 [arXiv:2103.01214] [INSPIRE].

[53] T. Cridge, M.A. Lim and R. Nagar, Wr production at NNLO+PS accuracy in GENEVA, arXiv:2105.13214 [INSPIRE].

[54] J. Gaunt, M. Stahlhofen, F.J. Tackmann and J.R. Walsh, N-jettiness Subtractions for NNLO QCD Calculations, JHEP 09 (2015) 058 [arXiv: 1505.04794] [INSPIRE].

[55] R. Boughezal et al., Color singlet production at NNLO in MCFM, Eur. Phys. J. C 77 (2017) 7 [arXiv: 1605.08011] [INSPIRE].

[56] J. Campbell and T. Neumann, Precision Phenomenology with MCFM, JHEP 12 (2019) 034 [arXiv: 1909.09117] [INSPIRE].

[57] G. Billis, M.A. Ebert, J.K.L. Michel and F.J. Tackmann, A toolbox for $q_{T}$ and 0-jettiness subtractions at $N^{3} L O$, Eur. Phys. J. Plus 136 (2021) 214 [arXiv:1909.00811] [INSPIRE].

[58] ATLAS collaboration, Measurement of event-shape observables in $Z \rightarrow \ell^{+} \ell^{-}$events in $p p$ collisions at $\sqrt{s}=7 \mathrm{TeV}$ with the ATLAS detector at the LHC, Eur. Phys. J. C 76 (2016) 375 [arXiv: 1602.08980] [INSPIRE]. 
[59] S. Fleming, A.H. Hoang, S. Mantry and I.W. Stewart, Jets from massive unstable particles: Top-mass determination, Phys. Rev. D 77 (2008) 074010 [hep-ph/0703207] [InSPIRE].

[60] S. Fleming, A.H. Hoang, S. Mantry and I.W. Stewart, Top Jets in the Peak Region: Factorization Analysis with NLL Resummation, Phys. Rev. D 77 (2008) 114003 [arXiv:0711.2079] [INSPIRE].

[61] B. Bachu, A.H. Hoang, V. Mateu, A. Pathak and I.W. Stewart, Boosted top quarks in the peak region with NL3L resummation, Phys. Rev. D 104 (2021) 014026 [arXiv:2012.12304] [INSPIRE].

[62] I.W. Stewart, F.J. Tackmann and W.J. Waalewijn, The Quark Beam Function at NNLL, JHEP 09 (2010) 005 [arXiv: 1002.2213] [InSPIRE].

[63] J.R. Gaunt, M. Stahlhofen and F.J. Tackmann, The Quark Beam Function at Two Loops, JHEP 04 (2014) 113 [arXiv:1401.5478] [InSPIRE].

[64] C.F. Berger, C. Marcantonini, I.W. Stewart, F.J. Tackmann and W.J. Waalewijn, Higgs Production with a Central Jet Veto at NNLL+NNLO, JHEP 04 (2011) 092 [arXiv: 1012.4480] [INSPIRE].

[65] J. Gaunt, M. Stahlhofen and F.J. Tackmann, The Gluon Beam Function at Two Loops, JHEP 08 (2014) 020 [arXiv: 1405.1044] [INSPIRE].

[66] M.A. Ebert, B. Mistlberger and G. Vita, $N$-jettiness beam functions at $N^{3} L O$, JHEP 09 (2020) 143 [arXiv: 2006.03056] [INSPIRE].

[67] R. Kelley, M.D. Schwartz, R.M. Schabinger and H.X. Zhu, The two-loop hemisphere soft function, Phys. Rev. D 84 (2011) 045022 [arXiv:1105.3676] [INSPIRE].

[68] P.F. Monni, T. Gehrmann and G. Luisoni, Two-Loop Soft Corrections and Resummation of the Thrust Distribution in the Dijet Region, JHEP 08 (2011) 010 [arXiv:1105.4560] [INSPIRE].

[69] L. Chen, M. Czakon and R. Poncelet, Polarized double-virtual amplitudes for heavy-quark pair production, JHEP 03 (2018) 085 [arXiv:1712.08075] [INSPIRE].

[70] T.T. Jouttenus, I.W. Stewart, F.J. Tackmann and W.J. Waalewijn, The Soft Function for Exclusive N-Jet Production at Hadron Colliders, Phys. Rev. D 83 (2011) 114030 [arXiv: 1102.4344] [INSPIRE].

[71] T. Becher and M. Neubert, Threshold resummation in momentum space from effective field theory, Phys. Rev. Lett. 97 (2006) 082001 [hep-ph/0605050] [INSPIRE].

[72] T. Becher, A. Broggio and A. Ferroglia, Introduction to Soft-Collinear Effective Theory, vol. 896, Springer (2015), [DOI] [arXiv:1410.1892] [INSPIRE].

[73] A.J. Buras, M. Jamin, M.E. Lautenbacher and P.H. Weisz, Effective Hamiltonians for $\Delta S=1$ and $\Delta B=1$ nonleptonic decays beyond the leading logarithmic approximation, Nucl. Phys. $B$ 370 (1992) 69 [Addendum ibid. 375 (1992) 501] [INSPIRE].

[74] G. Buchalla, A.J. Buras and M.E. Lautenbacher, Weak decays beyond leading logarithms, Rev. Mod. Phys. 68 (1996) 1125 [hep-ph/9512380] [INSPIRE].

[75] J. Butterworth et al., PDF4LHC recommendations for LHC Run II, J. Phys. G 43 (2016) 023001 [arXiv: 1510.03865] [INSPIRE].

[76] A. Buckley et al., LHAPDF6: parton density access in the LHC precision era, Eur. Phys. J. C 75 (2015) 132 [arXiv: 1412.7420] [INSPIRE]. 
[77] Z. Ligeti, I.W. Stewart and F.J. Tackmann, Treating the $b$ quark distribution function with reliable uncertainties, Phys. Rev. D 78 (2008) 114014 [arXiv:0807.1926] [INSPIRE].

[78] R. Abbate, M. Fickinger, A.H. Hoang, V. Mateu and I.W. Stewart, Thrust at $N^{3} L L$ with Power Corrections and a Precision Global Fit for $\alpha_{s}(m Z)$, Phys. Rev. D 83 (2011) 074021 [arXiv: 1006.3080] [INSPIRE].

[79] I.W. Stewart, F.J. Tackmann, J.R. Walsh and S. Zuberi, Jet $p_{T}$ resummation in Higgs production at $N N L L^{\prime}+N N L O$, Phys. Rev. D 89 (2014) 054001 [arXiv:1307.1808] [INSPIRE].

[80] M.A. Ebert and F.J. Tackmann, Impact of isolation and fiducial cuts on $q_{T}$ and $N$-jettiness subtractions, JHEP 03 (2020) 158 [arXiv:1911.08486] [INSPIRE].

[81] M.A. Ebert, J.K.L. Michel, I.W. Stewart and F.J. Tackmann, Drell-Yan $q_{T}$ resummation of fiducial power corrections at $N^{3} L L$, JHEP 04 (2021) 102 [arXiv:2006.11382] [INSPIRE].

[82] G. Bell, R. Rahn and J. Talbert, Two-loop anomalous dimensions of generic dijet soft functions, Nucl. Phys. B 936 (2018) 520 [arXiv:1805.12414] [INSPIRE].

[83] G. Bell, R. Rahn and J. Talbert, Generic dijet soft functions at two-loop order: correlated emissions, JHEP 07 (2019) 101 [arXiv: 1812.08690] [INSPIRE].

[84] G. Bell, R. Rahn and J. Talbert, Generic dijet soft functions at two-loop order: uncorrelated emissions, JHEP 09 (2020) 015 [arXiv: 2004.08396] [INSPIRE].

[85] A. Broggio, A. Ferroglia, B.D. Pecjak, A. Signer and L.L. Yang, Associated production of a top pair and a Higgs boson beyond NLO, JHEP 03 (2016) 124 [arXiv:1510.01914] [INSPIRE].

[86] A. Broggio, A. Ferroglia, G. Ossola and B.D. Pecjak, Associated production of a top pair and a $W$ boson at next-to-next-to-leading logarithmic accuracy, JHEP 09 (2016) 089 [arXiv: 1607.05303] [INSPIRE].

[87] A. Broggio, A. Ferroglia, B.D. Pecjak and L.L. Yang, NNLL resummation for the associated production of a top pair and a Higgs boson at the LHC, JHEP 02 (2017) 126 [arXiv: 1611.00049] [INSPIRE].

[88] A. Broggio, A. Ferroglia, G. Ossola, B.D. Pecjak and R.D. Sameshima, Associated production of a top pair and a Z boson at the LHC to NNLL accuracy, JHEP 04 (2017) 105 [arXiv: 1702.00800] [INSPIRE].

[89] C.W. Bauer, D. Pirjol and I.W. Stewart, Soft collinear factorization in effective field theory, Phys. Rev. D 65 (2002) 054022 [hep-ph/0109045] [INSPIRE].

[90] G. Somogyi, Angular integrals in d dimensions, J. Math. Phys. 52 (2011) 083501 [arXiv:1101.3557] [INSPIRE].

[91] V.E. Lyubovitskij, F. Wunder and A.S. Zhevlakov, New ideas for handling of loop and angular integrals in D-dimensions in QCD, JHEP 06 (2021) 066 [arXiv:2102.08943] [INSPIRE]. 